Provided for non-commercial research and education use. Not for reproduction, distribution or commercial use.

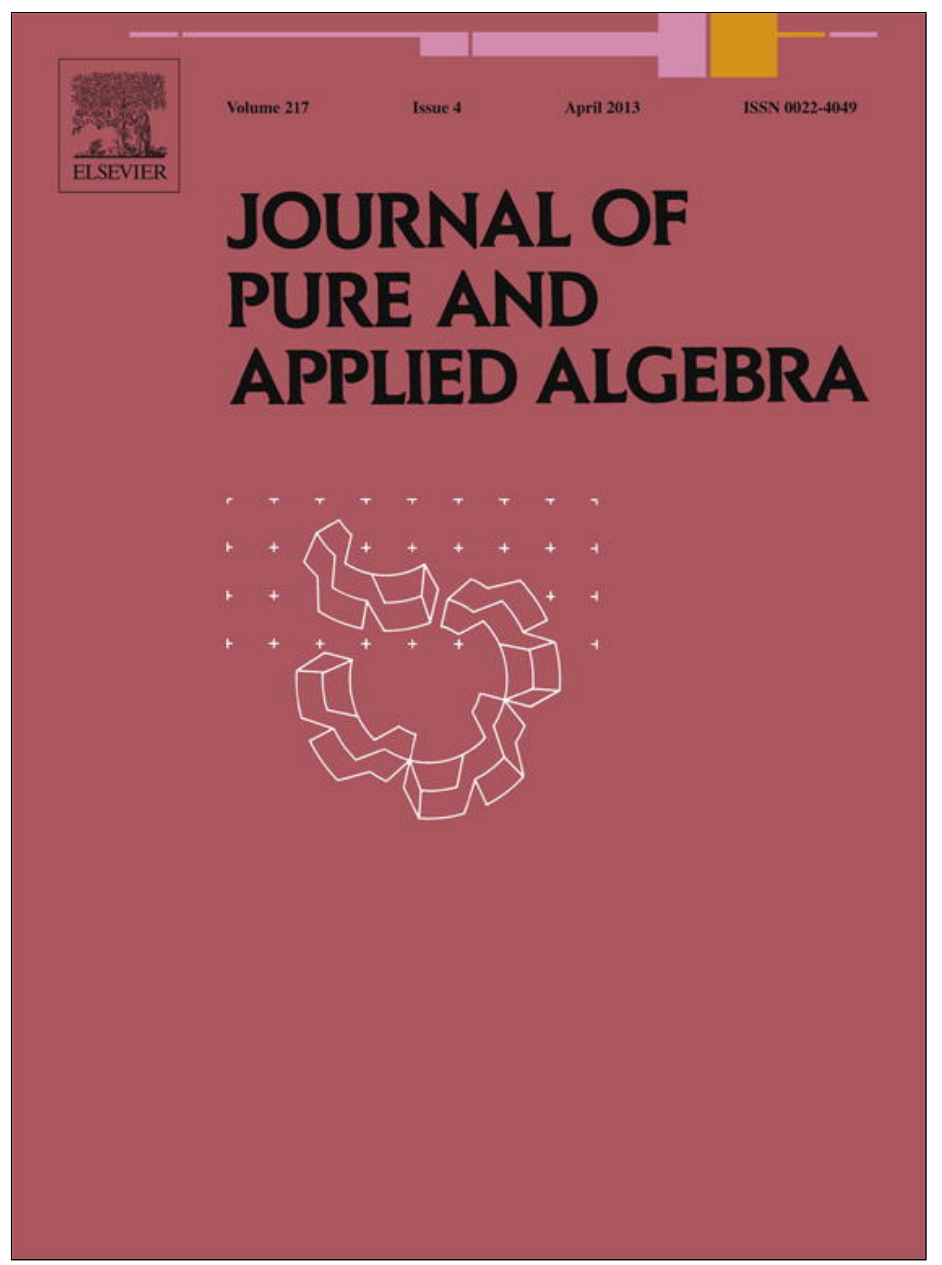

This article appeared in a journal published by Elsevier. The attached copy is furnished to the author for internal non-commercial research and education use, including for instruction at the authors institution and sharing with colleagues.

Other uses, including reproduction and distribution, or selling or licensing copies, or posting to personal, institutional or third party websites are prohibited.

In most cases authors are permitted to post their version of the article (e.g. in Word or Tex form) to their personal website or institutional repository. Authors requiring further information regarding Elsevier's archiving and manuscript policies are encouraged to visit:

http://www.elsevier.com/copyright 


\title{
Complete intersections in rational homotopy theory
}

\author{
J.P.C. Greenlees ${ }^{\mathrm{a}, *}$, K. Hess ${ }^{\mathrm{b}}$, S. Shamir ${ }^{\mathrm{c}}$ \\ a School of Mathematics and Statistics, Hicks Building, Sheffield S3 7RH, UK \\ ${ }^{\mathrm{b}}$ EPFL, Lausanne, Switzerland \\ ${ }^{c}$ Department of Mathematics, University of Bergen, 5008 Bergen, Norway
}

\section{A R T I C L E I N F O}

\section{Article history:}

Received 12 June 2009

Received in revised form 29 June 2012

Available online 7 September 2012

Communicated by S. Iyengar

\begin{abstract}
A B S T R A C T
We investigate various homotopy invariant formulations of commutative algebra in the context of rational homotopy theory. The main subject is the complete intersection condition, where we show that a growth condition implies a structure theorem and that modules have multiply periodic resolutions.
\end{abstract}

(C) 2012 Published by Elsevier B.V.

MSC: 55P62; $13 C 40$

\section{Introduction}

\subsection{Background}

It has been very fruitful to adapt the definitions of commutative algebra so that they apply in homotopy theory. The original motivation is that it is useful to study a space $X$ through a ring of functions, and for our purposes we will think of the ring $C^{*}(X ; k)$ of cochains on $X$. Of course, if the analogy is to be accurate, we need a commutative model for $C^{*}(X ; k)$, and if it is to be effective we need to render the definitions homotopy invariant.

The prime example of this is the connection between rational homotopy theory and rational differential graded algebras (DGAs), but the availability of good models for ring spectra has led to other useful examples in positive characteristic. The emphasis in classical rational homotopy theory has been on finite complexes and calculation, whereas one of the themes in characteristic $p$ has been to consider classifying spaces of compact Lie groups where the natural finiteness condition is that the cohomology rings in question are Noetherian. The purpose of the present paper is to take the ideas developed for compact Lie groups and investigate them in the much more accessible context of rational homotopy theory. From one point of view this is the process of generalizing classical results [11] from the case when $H^{*}(X ; \mathbb{Q})$ is finite dimensional to the case when it is Noetherian, and given the available tools of rational homotopy theory, this is reasonably straightforward. From another point of view this is an opportunity to give new and accessible examples of the theory, and to test expectations in a context where complete calculation is often possible. Finally, the work suggests a number of questions we may translate through the mirror [4] to local algebra, and we plan to investigate these in future.

\subsection{Contents}

On the commutative algebra side we restrict attention to commutative, local, Noetherian rings. On the topological side, we restrict attention to simply connected, rational spaces $X$ with $H^{*}(X)$ Noetherian.

\footnotetext{
* Corresponding author.

E-mail addresses: j.greenlees@sheffield.ac.uk (J.P.C. Greenlees), kathryn.hess@epfl.ch (K. Hess), shoham.shamir@math.uib.no (S. Shamir).
} 
We begin by considering analogues of regular local rings. This is already well-known in rational homotopy theory, but it gives us an opportunity to introduce some terminology and to express things in a convenient language. The regular spaces $X$ are precisely those which are finite products of even Eilenberg-MacLane spaces.

The main subject of the paper is a study of the complete intersection (ci) condition. We give a number of homotopy invariant definitions of ci spaces, corresponding to different aspects of the ci condition. Each definition will be indicated by an additional letter to indicate its character; although we will prove some of them equivalent in particular contexts, this device gives conceptual clarity, and it is essential in other contexts where the notions are not equivalent.

The definitions have very different characters, so it is striking that we are able to show that in the rational context several are equivalent. The structural condition in commutative algebra is that a ci ring is a quotient of a regular local ring by a regular sequence. We say that a simply connected rational space $X$ is sci if it is formed from a finite product of even EilenbergMacLane spaces by iterated spherical fibrations (all definitions are given precisely in Section 6). Second, Benson and the first author observed that Eisenbud's eventually multiperiodic resolutions [9] can be formulated as a finiteness condition (zci) on the derived category which is a strengthening of the condition in [8], using elements of the centre of the derived category. In the context of rational DGAs the centre is sometimes not large enough, so we need to relax the zci condition to a condition we call the eci condition, where e refers to endomorphisms of bimodules. Finally, there is a characterization in terms of growth. In commutative algebra the condition is that $\operatorname{Ext}_{R}^{*}(k, k)$ has polynomial growth (see Definition 9.1); the condition on a rational simply connected space is the growth condition (gci) that $H_{*}(\Omega X)$ has polynomial growth.

In commutative algebra the four conditions are equivalent for complete local rings: the sci condition implies the zci condition by Eisenbud's resolutions [9], the zci condition (or the weaker eci condition) implies the gci condition by elementary estimates on the growth of cohomology and the gci condition implies the sci condition by Gulliksen's theorem [12].

For simply connected rational DGAs we shall see that the sci implies eci and eci implies gci. We can show that under an additional finiteness hypothesis both implications can be reversed: this is the strongly Noetherian condition of Definition 3.5. Even in the presence of the strongly Noetherian condition, the zci condition is strictly stronger. Most remarkable of the equivalences is the fact that the growth condition implies a structure theorem: a strongly Noetherian space $X$ is gci if and only if there is a fibration

$$
F \longrightarrow X \longrightarrow K V
$$

where $K V$ is a finite product of even Eilenberg-MacLane spaces and $\pi_{*}(F)$ is finite dimensional and entirely in odd degrees. Amongst these spaces, those in which $F$ has trivial $k$-invariants, so that $F$ is a product of odd spheres, are the ones with pure Sullivan models.

We were struck by the radical effect of the Noetherian condition. On the one hand, an iterated spherical fibration over a product of even Eilenberg-MacLane spaces obviously has Noetherian cohomology. On the other hand we may investigate what happens if we drop the Noetherian condition in the gci and eci conditions. In the first case, we simply require $H_{*}(\Omega X)$ to have polynomial growth without requiring $H^{*}(X)$ to be Noetherian, and the Milnor-Moore theorem shows that this is equivalent to asking that $\pi_{*}(X)$ is finite dimensional. In the second case we obtain a certain evs condition (an endomorphism type condition related to the virtual smallness of [8]), which states that a suitable series of iterated mapping cones gives a small object. These two non-Noetherian conditions are also equivalent.

Altogether, we were surprised by the way the Noetherian condition affected behaviour, and we are grateful to N.P. Strickland for a timely remark. We also thank S.B. Iyengar and the referee for numerous comments, and detailed pointers to the literature, especially for the examples. Finally, we are grateful to Don Stanley, who constructed Example 8.7 highlighting a mistake in an earlier version.

\subsection{The layout of the paper}

After summarizing conventions in Section 2, we begin in Section 3 by giving a brief summary of the results and terminology we need from rational homotopy theory. We are then in a position to consider rational DGAs in parallel with rational spaces. In a series of sections we describe the definitions for rational DGAs and in particular for Sullivan models of rational spaces. In Section 4 we consider regular rings and spaces.

From Section 5 onwards, our main concern is for complete intersections. First, Section 5 discusses the centre of a derived category, and how bimodules and Hochschild cohomology give elements of the centre. Section 6 introduces the definitions designed to capture various aspects of hypersurface and ci spaces, which later sections show to be equivalent. Section 7 takes the structural definition, and shows that any sci space has a standard form. To give proper roots to our discussion, Section 8 gives a number of explicit examples illustrating the phenomena, and shows that the various classes of spaces are distinct. Returning to the proofs, Section 9 gives the elementary argument that eci spaces satisfy the gci growth condition. Section 10 shows that sci spaces all have eventually multiply periodic module theories. In Section 11 we calculate the Hochschild cohomology of all pure sci spaces relative to their regular base and use the result to show they are zci. Finally, and perhaps most interestingly, in Section 12 we show that the growth condition alone is enough to show that a space has the standard sci form. The final section explores the role of the Noetherian condition further, and gives a characterization of the polynomial growth of $H_{*}(\Omega X)$ when we do not require $H^{*}(X)$ to be Noetherian in the same style as the multiply periodic resolution property for ci spaces. 


\section{Conventions}

\subsection{Terminology for triangulated categories}

Recall that an object $X$ of a triangulated category $\mathcal{T}$ is called small if the natural map

$$
\bigoplus_{i}\left[X, Y_{i}\right] \longrightarrow\left[X, \bigvee_{i} Y_{i}\right]
$$

is an isomorphism for any set of objects $Y_{i}$.

A thick subcategory of $\mathcal{T}$ is a full subcategory closed under completion of triangles and taking retracts. We write thick $(X)$ for the smallest thick subcategory containing $X$, and if $A \in \operatorname{thick}(X)$ we also say ' $X$ finitely builds $A$ ' and write $X \models A$.

A localizing subcategory of $\mathcal{T}$ is a thick subcategory which is also closed under taking arbitrary coproducts. We write $\operatorname{loc}(X)$ for the smallest localizing subcategory containing $X$, and if $A \in \operatorname{loc}(X)$ we also say ' $X$ builds $A$ ' and write $X \vdash A$.

Following [8] we say that $X$ is virtually small if thick $(X)$ contains a non-trivial small object $W$, and we say that any such $W$ is a witness for the fact that $X$ is virtually small.

\subsection{Grading conventions}

We will have cause to discuss homological and cohomological gradings. Our experience is that this a frequent source of confusion, so we adopt the following conventions. First, we refer to lower gradings as degrees and upper gradings as codegrees. As usual, one may convert gradings to cogradings via the rule $M_{n}=M^{-n}$. Thus both chain complexes and cochain complexes have differentials of degree -1 (which is to say, of codegree 1 ). This much is standard. However, since we need to deal with both chain complexes and cochain complexes it is essential to have separate notation for homological suspensions $\left(\Sigma^{i}\right)$ and cohomological suspensions $\left(\Sigma_{i}\right)$ : these are defined by

$$
\left(\Sigma^{i} M\right)_{n}=M_{n-i} \text { and }\left(\Sigma_{i} M\right)^{n}=M^{n-i} .
$$

Thus, for example, with reduced chains and cochains of a based space $X$, we have

$$
\tilde{C}_{*}\left(\Sigma^{i} X\right)=\Sigma^{i} \tilde{C}_{*}(X) \text { and } \tilde{C}^{*}\left(\Sigma^{i} X\right)=\Sigma_{i} \tilde{C}^{*}(X) .
$$

\subsection{Other conventions}

Unless explicitly stated to the contrary, all coefficients will be in the rational numbers $\mathbb{Q}$, and for a rational vector space $V$, we write $V^{\vee}=\operatorname{Hom}_{\mathbb{Q}}(V, \mathbb{Q})$ for the dual vector space.

For brevity we write CGA for commutative graded algebra (i.e., an algebra which is commutative in the graded sense that $\left.x y=(-1)^{|x| \cdot|y|} y x\right)$, DGA for differential graded algebra, and CDGA for commutative differential graded algebra. When we refer to modules over a DGA, we intend differential graded modules unless otherwise stated.

Finally, for a space $X$, we write $C^{*}(X)$ for an unspecified CDGA model for the cochains on $X$. In keeping with our philosophy of making homotopy invariant constructions, we usually write $\otimes$ for the left derived tensor product.

\section{Rational homotopy theory}

Rational homotopy theory provides the ideal context to test ideas about homotopy invariant commutative algebra. On the one hand many aspects of commutative algebra are especially simple for $\mathbb{Q}$-algebras and on the other we can appeal to the intuition and structures of homotopy theory.

In this section we describe our conventions, thereby recalling standard material (see [11] for a much fuller development).

\subsection{Terminology for commutative differential graded algebras}

We will restrict attention to simply connected $\mathbb{Q}$-algebras of finite type.

If $V$ is a graded rational vector space, we write $\Lambda(V)$ for the free CGA on $V$. This is a symmetric algebra on $V^{e v}$ tensored with an exterior algebra on $V^{\text {od }}$. A Sullivan algebra is a CDGA which is free as a CGA on a simply connected graded vector space $V$ of finite dimension in each degree, and whose differential has the property that if $x \in V^{s}$ then $d x \in \Lambda\left(V^{<s}\right)$. It is minimal if in addition $d$ takes values in $\Lambda^{+}(V)^{2}$. A Sullivan algebra $\left(\Lambda\left(V^{o d} \oplus V^{e v}\right), d\right)$ is pure if $d\left(V^{o d}\right) \subset \Lambda V^{e v}$ and $d\left(V^{e v}\right)=0$.

A relative Sullivan algebra is a map $M \longrightarrow M \rtimes \Lambda(V)$ of CDGAs. Here the underlying CGA of $M \rtimes \Lambda(V)$ is $M \otimes \Lambda(V)$, and the notation expresses the fact that $M$ is a sub-DGA and $\Lambda(V)$ is a quotient.

\subsection{Rational models for simply connected spaces}

Any simply connected rational CW-complex with cohomology finite in each degree is modelled by a simply connected rational CDGA (such as the CDGA of PL polynomial differential forms $\mathscr{A}_{P L}(X)$ ). Furthermore, any such CDGA has a Sullivan 
minimal model, unique up to isomorphism. We write $C^{*}(X)$ for an unspecified CDGA model for the cochains on $X$. The process of building up a Sullivan algebra degree by degree corresponds to building up a space using a Postnikov tower.

If $V$ is an evenly graded vector space, we write $K V$ for the associated Eilenberg-MacLane space. In principle we could use the same notation when $V$ has an odd summand, but we will not do so. Since odd spheres are rational Eilenberg-MacLane spaces, if $W$ is a graded vector space in odd degrees, we write $S(W)$ for the corresponding Eilenberg-MacLane space.

A fibration $E \longrightarrow B$ with fibre $F$ can be modelled by a relative Sullivan algebra $M \rtimes \Lambda(V) \longleftarrow M$ where $M$ models $B$, $M \rtimes \Lambda(V)$ models $E$ and the fibre $F$ is then modelled by $\Lambda(V)$.

\subsection{Homotopy Lie algebras and the Milnor-Moore theorem}

Recall that $\pi_{*}(\Omega X)$ is a graded Lie algebra under the Samelson product. This is connected to the homology via the universal enveloping algebra.

Theorem 3.1 (Milnor-Moore [15]). If X is a simply connected rational space then

$$
H_{*}(\Omega X)=U\left(\pi_{*}(\Omega X)\right) .
$$

The Poincaré-Birkhoff-Witt theorem states that if we filter $U(L)$ by tensor length then there is an isomorphism $\operatorname{Gr}(U(L))=\Lambda L$. In particular, when $L$ is finite dimensional the dimensions of the homogeneous pieces of $U(L)$ are the same as that of the symmetric algebra on $L^{e v}$. We see that if $H^{*}(X)$ is Noetherian $H_{*}(\Omega X)$ is of polynomial growth if and only if $\pi_{*}(\Omega X)$ is finite dimensional, and in that case the growth is bounded by a polynomial of degree one less than

$$
\operatorname{dim}_{\mathbb{Q}}\left(\pi_{e v}(\Omega X)\right)=\operatorname{dim}_{\mathbb{Q}}\left(\pi_{o d}(X)\right) .
$$

This is also a consequence of Corollary 3.3.

\subsection{Elliptic spaces}

Perhaps for historical reasons, classical rational homotopy theory concentrates on finite complexes, which is to say spaces with $H^{*}(X)$ finite dimensional. These correspond to 0 -dimensional local rings.

A simply connected rational space $X$ is called elliptic if $H^{*}(X)$ and $\pi_{*}(X)$ are both finite dimensional. It is called hyperbolic if $\pi_{*}(X)$ has exponential growth.

A major theorem of rational homotopy theory is the Dichotomy Theorem stating that a simply connected rational space with $H^{*}(X)$ finite dimensional is either elliptic or hyperbolic. In a sense we will make precise, elliptic spaces correspond to 0-dimensional complete intersections.

\subsection{Noether normalization}

Polynomial rings on even degree generators play a special role in the theory. To start with, they are intrinsically formal: if $P$ is a polynomial ring on even degree generators, then if $A$ is any CDGA with $H^{*}(A) \cong P$, we have a quasi-isomorphism $A \simeq P$. Indeed, $P$ has a useful universal property: for any CDGA $A$, and any map $\theta: P \longrightarrow H^{*}(A)$ of CGAs, a choice of representative cycles for the polynomial generators allows us to realize $\theta$ by a map $\tilde{\theta}: P \longrightarrow A$ of CDGAs. Not only are they convenient, we shall see they have a structural role: polynomial rings on even degree generators provide the class of CDGAs corresponding to regular local rings. We think of $K V$ with $V$ even and finite dimensional as a generalization of the rational classifying space of a compact connected Lie group.

Polynomial rings can then be used in the study of general Noetherian rings. Indeed, the Noether normalization theorem states that if $R$ is a Noetherian connected CGA, it is finitely generated as a module over a polynomial subalgebra $P$ on even degree generators. We will repeatedly use the following counterpart of this statement.

Proposition 3.2. If $X$ is a 1-connected rational space with $H^{*}(X)$ Noetherian, there is a fibration

$$
F \longrightarrow X \longrightarrow K V
$$

of rational spaces where $V$ is even and finite dimensional, and $H^{*}(F)$ is finite dimensional.

Proof. By Noether normalization, $H^{*}(X)$ is finitely generated as a module over a polynomial algebra $P$ on even degree generators. Choosing representative cycles, we have a map $P=K V \longrightarrow C^{*}(X)$ of CDGAs realizing this map in cohomology. This gives a fibration

$$
F \longrightarrow X \longrightarrow K V \text {. }
$$

To see $H^{*}(F)$ is finite dimensional, we note that $H^{*}(X)$ is a finitely generated $P$-module, and therefore has a finite resolution by finitely generated free $P$-modules. 
We refer to this fibration as a Noether normalization of $X$, and to $F$ as a Noether fibre of $X$. The long exact sequence in homotopy shows that the growth of $\pi_{*}(X)$ is the same as that of $\pi_{*}(F)$, so that the classical Dichotomy Theorem translates directly to our context.

Corollary 3.3 (Dichotomy). For a space X with $H^{*}(X)$ Noetherian, either $\pi_{*}(X)$ is finite dimensional or it has exponential growth. The homotopy is finite dimensional if and only if a Noether fibre is elliptic.

This motivates the following extension of the notion of elliptic spaces to spaces with Noetherian cohomology.

Definition 3.4. A space $X$ is gci (or satisfies the growth condition for a complete intersection) if $H^{*}(X)$ is Noetherian and $\pi_{*}(X)$ is finite dimensional.

These spaces are the principal subject of the present paper, and we return to them in Section 6.

Unfortunately the Noetherian condition does not have the hereditary properties we require, and we will need a stronger condition. For this we need to extend the notion of homotopy groups to a Sullivan algebra $(\Lambda V, d)$ : here $\pi_{*}(\Lambda V, d)$ is the dual of the vector space $H^{*}\left(V ; d_{1}\right)$, where $d_{1}$ is the linear part of the differential $d$. Thus if $(\Lambda V, d)$ is minimal, the homotopy groups are dual to $V$.

Definition 3.5. We say that $X$ is strongly Noetherian if every sub-DGA of its minimal model whose inclusion induces a monomorphism of dual homotopy groups has Noetherian cohomology.

Remark 3.6. The space $X$ is strongly Noetherian if and only if for every Sullivan extension $(\Lambda V, d) \longrightarrow(\Lambda V \otimes \Lambda W, D) \simeq$ $C^{*}(X)$ that has the property that $\left(V, d_{1}\right) \longrightarrow\left(V \oplus W, D_{1}\right)$ induces a monomorphism in cohomology, $H^{*}(\Lambda V, d)$ is Noetherian.

\section{Regular rings and spaces}

We shall show that the regular spaces are precisely the spaces $K V$ where $V$ is even and finite dimensional. This is straightforward once we have established definitions.

For all classical commutative algebras, we refer the reader to [14].

\subsection{Definitions}

In commutative algebra there are three styles for a definition of a regular local ring: a version in terms of sequences, in terms of the growth of the Ext algebra and one in terms of modules.

Definition 4.1. (i) A local Noetherian ring $R$ is $s$-regular if the maximal ideal is generated by a regular sequence.

(ii) A local Noetherian ring $R$ is $g$-regular if $\operatorname{Ext}_{R}^{*}(k, k)$ is finite dimensional.

(iii) A local Noetherian ring $R$ is m-regular if every finitely generated module is small in $\mathbf{D}(R)$.

It is not hard to see that g-regularity is equivalent to m-regularity or that s-regularity implies g-regularity. Serre proved that g-regularity implies s-regularity, so the three conditions are equivalent.

It is not altogether clear what should play the role of finitely generated modules in the more general context. We would like it to include all small objects, and the object $\mathbb{Q}$, and we would like to know that if $\mathbb{Q}$ is small then all "finitely generated" objects are small. For the purpose of the present paper, we take

$$
\mathcal{F} g:=\left\{M \mid H^{*}(M) \text { is a finitely generated } H^{*}(X) \text {-module }\right\},
$$

and we will show that it has the properties we require.

Definition 4.2. (i) A space $X$ is s-regular if there are fibrations

$$
S^{n_{1}} \longrightarrow X_{1} \longrightarrow X, S^{n_{2}} \longrightarrow X_{2} \longrightarrow X_{1}, \ldots, S^{n_{d}} \longrightarrow X_{d} \longrightarrow X_{d-1}
$$

with $X_{d} \simeq *$.

(ii) A space $X$ is $g$-regular if $H_{*}(\Omega X)$ is finite dimensional.

(iii) A space $X$ is $m$-regular if every object of $\mathcal{F} g$ is small in $\mathbf{D}\left(C^{*}(X)\right)$.

If $X$ is s-regular, we see $\Omega X_{d-1} \simeq S^{n_{d}}$, and working back up the sequence of fibrations, we see that $X$ is g-regular. Since $\mathbb{Q} \in \mathcal{F} g$ it follows from the Eilenberg-Moore Theorem that an m-regular space is g-regular. We will establish the reverse implication by classifying g-regular spaces.

\subsection{Classification of regular spaces}

In the rational context we can give a complete classification of regular spaces.

Theorem 4.3. A simply connected rational space $X$ of finite type is g-regular if and only if $\pi_{*}(X)$ is even and finite dimensional. It is therefore equivalent to the Eilenberg-MacLane space $K\left(\pi_{*}(X)\right)$, and has polynomial cohomology $\operatorname{Symm}\left(\pi_{*}(X)\right)$.

Proof. Since $\Omega X$ is a product of Eilenberg-MacLane spaces, we need only remark that odd Eilenberg-MacLane spaces are spheres, whereas even Eilenberg-MacLane spaces are infinite dimensional. 
Proposition 4.4. If $X$ is g-regular and $H^{*}(M)$ is finitely generated over $H^{*}(X)$ then $M$ is small.

Proof. Suppose $H^{*}(M)$ is a finitely generated $H^{*}(X)$-module. Since $H^{*}(X)$ is a polynomial ring on even degree generators, there is a finite resolution by finitely generated free modules

$$
0 \longrightarrow P_{r} \stackrel{d_{r}}{\longrightarrow} P_{r-1} \stackrel{d_{r-1}}{\longrightarrow} \cdots \stackrel{d_{2}}{\longrightarrow} P_{1} \stackrel{d_{1}}{\longrightarrow} P_{0} \stackrel{d_{0}}{\longrightarrow} H^{*}(M) \longrightarrow 0
$$

We proceed to realize this in the usual way. To start with we realize the free modules $P_{i}=\left(H^{*}(X)\right)^{\oplus n}$ by the $C^{*}(X)$-modules $\mathbb{P}_{i}=\left(C^{*}(X)\right)^{\oplus n}$. Now take $M=M_{0}$ and realize the algebraic resolution by constructing a diagram

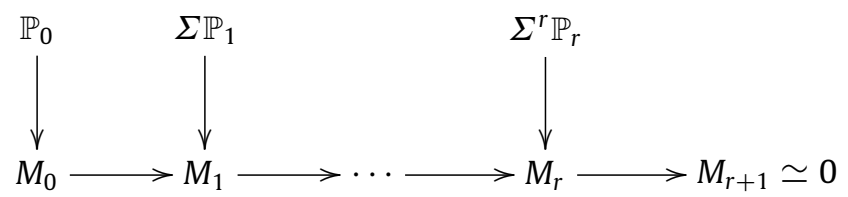

in which the sequences $\Sigma^{i} \mathbb{P}_{i} \longrightarrow M_{i} \longrightarrow M_{i+1}$ are cofibre sequences, $H^{*}\left(\Sigma^{-i} M_{i}\right)=\operatorname{ker}\left(d_{i-1}\right)$ and $\Sigma^{i} \mathbb{P}_{i} \longrightarrow M_{i}$ realizes the map in the algebraic resolution. Reversing the process, we see that $M_{r}, M_{r-1}, \ldots, M_{1}$ and $M_{0}=M$ are finitely built from $C^{*}(X)$ and therefore small as required.

This establishes the equivalence of the three definitions of regularity.

Corollary 4.5. A space is s-regular if and only if it is g-regular if and only if it is m-regular.

Remark 4.6. Since the proposition shows that finite generation is equivalent to a well-known condition for regular rings, it gives an alternative definition of the class $\mathcal{F} g$. Indeed we note that $\mathcal{F} g$ can be described as the class of all $R$-modules $M$ which are small as a $Q$-module, for some regular ring $Q$ and some ring map $Q \longrightarrow R$ making $R$ small as a $Q$-module. In other contexts where we do not have such a good understanding of objects, this seems the more convenient definition.

\subsection{Some small objects}

It is useful to identify some modules that are small rather generally.

Lemma 4.7. If $f: Y \longrightarrow X$ is a map with homotopy fibre $F(f)$ so that $H^{*}(F(f))$ is finite dimensional, then $C^{*}(Y)$ is small in $\mathbf{D}\left(C^{*}(X)\right)$.

Proof. By hypothesis, $\mathbb{Q}$ finitely builds $C^{*}(F(f))$ as a $C_{*}(\Omega X)$-module. Applying $\operatorname{Hom}_{C_{*}(\Omega X)}(\mathbb{Q}, \cdot)$, we deduce from the Eilenberg-Moore spectral sequence that $C^{*}(X)$ finitely builds $C^{*}(Y)$. In symbols,

$$
\mathbb{Q} \mid C_{*}(\Omega X) C^{*}(F(f))
$$

and hence

$$
C^{*}(X) \simeq \operatorname{Hom}_{C_{*}(\Omega X)}(\mathbb{Q}, \mathbb{Q}) \models \operatorname{Hom}_{C_{*}(\Omega X)}\left(\mathbb{Q}, C^{*}(F(f)) \simeq C^{*}(Y) .\right.
$$

\section{The centre of a triangulated category}

It will be useful to recall certain constructions before turning to complete intersections.

\subsection{Universal Koszul complexes}

To start with we suppose given a triangulated category $\mathcal{T}$. The centre $Z \mathcal{T}$ of $\mathcal{T}$ is defined to be the graded ring of graded endomorphisms of the identity functor.

Given $\chi \in Z \mathcal{T}$ of degree $a$, for any object $X$, we may form the mapping cone $X / \chi$ of $\chi: \Sigma^{a} X \longrightarrow X$. This is well defined up to non-unique equivalence. Indeed, given a map $f: X \longrightarrow Y$, the axioms of a triangulated category give a map $f: X / \chi \longrightarrow Y / \chi$ consistent with the defining triangles, but this is not usually unique or compatible with composition.

Now given a sequence of elements $\chi_{1}, \chi_{2}, \ldots, \chi_{n}$ we may iterate this construction, and form the Koszul complex

$$
K(X ; \chi):=X / \chi_{1} / \chi_{2} / \cdots / \chi_{n}
$$

it is universal in the sense that the construction applies uniformly to all objects $X$.

\subsection{Bimodules and the centre}

Bimodules provide a useful source of elements of $Z \mathbf{D}(R)$. Indeed, if $R$ is a flat $l$-algebra, and if $X \longrightarrow Y$ is a map of $R$ bimodules over $l$ (which is to say, of modules over $R^{e}=R \otimes_{l} R$ ), then for any $R$-module $M$ we obtain a map

$$
X \otimes_{R} M \longrightarrow Y \otimes_{R} M
$$

of $R$-modules, natural in $M$. 
It is sometimes convenient to package this in terms of the Hochschild cohomology ring

$$
H H^{*}(R \mid l)=\operatorname{Ext}_{R^{e}}^{*}(R, R) .
$$

If $l=\mathbb{Q}$, it is usual to omit it from the notation. Now a codegree $d$ element of this cohomology ring can be viewed as a map $R \longrightarrow \Sigma^{d} R$ in the category of $(R, R)$-bimodules, so that taking $X=Y=R$ above, we obtain a ring homomorphism

$$
H H^{*}(R \mid l) \longrightarrow Z \mathbf{D}(R) \text {. }
$$

If $R$ is an $l$-algebra which is not flat, $R^{e}=R \otimes_{l} R$ is taken in the derived sense, and similarly for $H H^{*}(R \mid l)$.

Given maps $l \longrightarrow Q \longrightarrow R$, we obtain a map $R \otimes_{l} R \longrightarrow R \otimes_{Q} R$ and hence a ring map $H H^{*}(R \mid Q) \longrightarrow H H^{*}(R \mid l)$. In particular, we have maps

$$
R=H H^{*}(R \mid R) \longrightarrow H H^{*}(R \mid Q) \longrightarrow H H^{*}(R \mid \mathbb{Q})=H H^{*}(R) .
$$

If $R=C^{*}(X)$, we may always take $l=\mathbb{Q}=C^{*}(p t)$, so that a bimodule is a module over $R^{e}=C^{*}(X \times X)$, but it is usually more appropriate to work over $Q=C^{*}(K)$ where we have a fibration $X \longrightarrow K$. In that case a bimodule over $Q$ is a module over $R^{e}=C^{*}\left(X \times_{K} X\right)$.

\subsection{Hochschild cohomology transcended}

It seems natural to relax the role of Hochschild cohomology. For us it is really just a tool for building bimodules from $R$. We will suppose given a map $Q \longrightarrow R$ so that $Q$ is regular and $R$ is small over $Q$. This ensures that $\mathcal{F} G$ as defined in Section 4 coincides with the $R$-modules which are small over $Q$.

Now, if $X$ is any $R$-bimodule finitely built from $R$, we can apply $\otimes_{R} M$ to deduce $X \otimes_{R} M$ is finitely built from $M=R \otimes_{R} M$ :

$$
R \models_{R^{e}} X \text { implies } M=R \otimes_{R} M \models_{R} X \otimes_{R} M .
$$

The important case for us is when $X$ is a small $R^{e}$-module.

Lemma 5.1. If $X$ is a small $R^{e}$-module and $M \in \mathcal{F} G$, then $X \otimes_{R} M$ is a small $R$-module.

Proof. It suffices to consider the case $X=R^{e}$. We then have

$$
X \otimes_{R} M=R \otimes_{\mathbb{Q}} R \otimes_{R} M=R \otimes_{\mathbb{Q}} M .
$$

By Proposition 4.4, $M$ is small as a $Q$-module, so

$$
R=R \otimes_{Q} Q \models R \otimes_{Q} M
$$

as required.

This comes close to saying that if $R$ is virtually small as an $R^{e}$-module then every $M \in \mathcal{F} g$ is virtually small as an $R$ module. The only obstacle is the need to show $X \otimes_{R} M$ is non-trivial; in the context we need it, the non-zero degree of the maps constructing $X$ will make it clear.

\section{Complete intersection rings and spaces}

We will give definitions of complete intersections as in the regular case. For commutative Noetherian rings these are known to be equivalent. We will investigate their relationship for rational spaces.

\subsection{The definition}

In commutative algebra there are three styles for a definition of a complete intersection ring: in structural terms, in terms of the growth of the Ext algebra and a version in terms of resolutions of modules.

Definition 6.1. (i) A local ring $R$ is an sci ring if $R=Q /\left(f_{1}, f_{2}, \ldots, f_{c}\right)$ for some regular ring $Q$ and some regular sequence $f_{1}, f_{2}, \ldots, f_{c}$. The minimum such $c$ (over all $Q$ and regular sequences) is called the codimension of $R$.

(ii) A local ring $R$ is $g c i$ if it is Noetherian and $\operatorname{Ext}_{R}^{*}(k, k)$ has polynomial growth. The $g$-codimension of $R$ is one more than the degree of the growth.

(iii) A local ring $R$ is $z c i$ if it is Noetherian and there are elements $z_{1}, z_{2}, \ldots z_{c} \in Z \mathbf{D}(R)$ of non-zero degree so that $M / z_{1} / z_{2} / \cdots / z_{c}$ is small for all finitely generated modules $M$. The minimum such $c$ is called the $z$-codimension of $R$.

The zci condition implies that every finitely generated module finitely builds a small complex in a prescribed manner using elements in $Z \mathbf{D}(R)$. One source of elements of $Z \mathbf{D}(R)$ is from Hochschild cohomology, or equivalently from bimodules. We can relax the zci condition by demanding only that each step in the building of the small complex is the cone of a bimodule endomorphism of the previous step. This is the essence of the next definition. 
(iv) A local ring $R$ is eci if it is Noetherian and there are a regular ring $Q$, a map $Q \longrightarrow R$ and homotopy cofibration sequences of $R^{e}$-modules, where $R^{e}=R \otimes_{\mathbb{Q}} R$,

$$
R=M_{0} \stackrel{g_{1}}{\rightarrow} \Sigma^{n_{1}} M_{0} \rightarrow M_{1}, \ldots, M_{c-1} \stackrel{g_{c}}{\rightarrow} \Sigma^{n_{c}} M_{c-1} \rightarrow M_{c}
$$

such that $M_{c}$ is small as an $R^{e}$-module and the degree of each $g_{i}$ is not zero.

Remark 6.2. The zci condition may be viewed as a coherent refinement of the quasi-complete intersection (qci) condition of [8], which states that every finitely generated object is virtually small.

Theorem 6.3 ([12,9]). For a complete local Noetherian ring the conditions sci, eci, gci and zci are all equivalent, and the corresponding codimensions are equal.

A construction of Shamash [17] shows that if $R$ is ci of codimension $c$, one may construct a free resolution which is eventually $c$-multiperiodic, and if the module is finitely generated, the terms are finitely generated. Furthermore, the resolution is constructed in [9] as a module over a polynomial ring on $c$ polynomial generators of degree -2 , which shows directly that $R$ is zci (the form of the resolution due to Avramov and Buchweitz [3] as described in [1, Section 9] makes this very clear). Considering the module $k$ shows that the ring $\operatorname{Ext}_{R}^{*}(k, k)$ has polynomial growth. The most striking result about ci rings is the theorem of Gulliksen [12] which states in the present language gci implies sci.

Remark 6.4. In commutative algebra, Avramov [2] proved Quillen's conjectured characterization of complete intersections by the fact that the André-Quillen cohomology is bounded. When $k$ is of characteristic 0 , the DG André-Quillen cohomology of $C^{*}(X)$ gives the dual homotopy groups of $X$, so the counterpart of Avramov's characterization is the gci condition.

On the other hand in positive characteristic, results of Mandell [13] show that the topological André-Quillen cohomology of $C^{*}(X)$ vanishes quite generally, so this does not give an appropriate counterpart of the ci condition.

\subsection{Definitions for spaces}

Adapting the above definitions for spaces is straightforward.

Definition 6.5. (i) A space $X$ is spherically $c i$ (sci) if it is formed from a regular space $K V$ using a finite number of spherical fibrations. More precisely, we require that there is a regular space $X_{0}=K V$ with $V$ even and finite dimensional, and fibrations

$$
S^{n_{1}} \longrightarrow X_{1} \longrightarrow X_{0}=K V, S^{n_{2}} \longrightarrow X_{2} \longrightarrow X_{1}, \ldots, S^{n_{c}} \longrightarrow X_{c} \longrightarrow X_{c-1}
$$

with $X=X_{c}$. The least such $c$ is called the $s$-codimension of $X$.

(ii) A space $X$ is a gci space if $H^{*}(X)$ is Noetherian and $H_{*}(\Omega X)$ has polynomial growth. The $g$-codimension of $X$ is one more than the polynomial degree of growth.

(iii) A space $X$ is a $z c i$ space if $H^{*}(X)$ is Noetherian and there are elements $z_{1}, z_{2}, \ldots, z_{c} \in Z \mathbf{D}\left(C^{*}(X)\right)$ of non-zero degree so that $C^{*}(Y) / z_{1} / z_{2} / \cdots / z_{c}$ is small for all $C^{*}(Y) \in \mathcal{F} g$. The minimum such $c$ is called the $z$-codimension of $X$.

(iv) A space $X$ is an eci space if $H^{*}(X)$ is Noetherian, there is a regular space $K$ and fibration $X \longrightarrow K$ with $C^{*}(X)$ small over $C^{*}(K)$ and there are homotopy cofibration sequences of $C^{*}\left(X \times_{K} X\right)$-modules,

$$
C^{*}(X)=M_{0} \stackrel{g_{1}}{\rightarrow} \Sigma^{n_{1}} M_{0} \rightarrow M_{1}, \ldots, M_{c-1} \stackrel{g_{c}}{\rightarrow} \Sigma^{n_{c}} M_{c-1} \rightarrow M_{c}
$$

such that $M_{c}$ is small as an $C^{*}\left(X \times_{K} X\right)$-module and the degree of each $g_{i}$ is not zero. The minimum such $c$ is called the e-codimension of $X$.

The main result of this paper is as follows.

Theorem 6.6. For a rational space $X$ we have the implications

$$
\mathrm{sci} \Rightarrow \mathrm{eci} \Rightarrow \mathrm{gci} \text {. }
$$

If the space is strongly Noetherian, the conditions are equivalent. If in addition $X$ is pure, then the conditions above are equivalent to the zci condition.

We will establish the implications

$$
\text { sci } \stackrel{A}{\Rightarrow} \text { eci } \stackrel{B}{\Rightarrow} \text { gci } \stackrel{C}{\Rightarrow} \text { sci. }
$$

We establish A in Section 10, B in Section 9, and under the additional hypothesis that $X$ is strongly Noetherian, we establish $\mathrm{C}$ in Section 12. The first two implications are fairly straightforward in the sense that they can also be proved in the nonrational context [5]. The implication $C$ takes a growth condition and gives a structure theorem, and could be viewed as the main result of the present paper. In Section 11 we show that a pure sci space is zci, while in Section 9 we show that the zci condition implies gci. 


\section{Standard form for sci spaces}

Of the conditions, the easiest to get a grip on is the sci condition, and it seems worthwhile to begin by anchoring it in reality by giving a structure theorem. In the rational context, we may put sci spaces into a standard form.

Theorem 7.1. A space $X$ is sci if and only if there exists a fibration sequence

$$
F \rightarrow X \rightarrow K V
$$

where $K V$ is a regular space and $\pi_{*}(F)$ is finite dimensional and entirely in odd degrees; in this case

$s-\operatorname{codim}(X)=\operatorname{dim}_{\mathbb{Q}}\left(\pi_{*}(F)\right)=\operatorname{dim}_{\mathbb{Q}}\left(\pi_{\text {odd }}(X)\right)$. odd.

Before proceeding it is useful to note that all the spherical fibrations in the definition of an sci space may be taken to be

Lemma 7.2. If $X$ can be formed from $B$ with an even spherical fibration $S^{2 m} \longrightarrow X \longrightarrow B$, then it can be formed from $B \times K(\mathbb{Q}, 2 m)$ by an odd spherical fibration

$$
S^{4 m-1} \longrightarrow X \longrightarrow B \times K(\mathbb{Q}, 2 m) \text {. }
$$

Accordingly, an sci space of s-codimension c may be constructed in c steps from a regular space using only odd dimensional spherical fibrations.

Proof. If $C^{*}(X)=C^{*}(B) \rtimes \Lambda\left(x_{2 m}, y_{4 m-1}\right)$, then if $d y=x^{2}+a x+b$ we may change basis by taking $x^{\prime}=x+a / 2$ and find $d x^{\prime}=0, d y=\left(x^{\prime}\right)^{2}+z$, where $z=b-a^{2} / 4 \in C^{*}(B)$. Adjoining $x^{\prime}$ to the model of $B$, we get the base of the required fibration.

Proof of Theorem 7.1. If $X$ is sci, by Lemma 7.2 we may use only odd spheres in the fibres. Now the composite function $X \longrightarrow K V$ has fibre with only odd dimensional homotopy, giving a fibration of the stated form.

We prove the converse statement by induction on the dimension of the odd homotopy. The result is trivial if the homotopy is entirely even. Suppose then that $X$ lies in a fibration

$$
F \longrightarrow X \longrightarrow K V
$$

and that $x \in \pi_{m}(F)$ is an element of highest degree. Construct a fibration

$$
S^{m} \longrightarrow F \longrightarrow F^{\prime}
$$

by killing $x$, so that $\operatorname{dim}_{\mathbb{Q}}\left(\pi_{*}\left(F^{\prime}\right)\right)=\operatorname{dim}_{\mathbb{Q}}\left(\pi_{*}(F)\right)-1$. Thus, we may choose models so that $C^{*}(F)=C^{*}\left(F^{\prime}\right) \rtimes C^{*}\left(S^{m}\right)$, and

$$
C^{*}(X)=C^{*}(K V) \rtimes\left[C^{*}\left(F^{\prime}\right) \rtimes C^{*}\left(S^{m}\right)\right] .
$$

Let $X^{\prime}$ be modelled by the subalgebra generated by $C^{*}(K V)$ and $C^{*}\left(F^{\prime}\right)$. This gives fibrations

$$
S^{m} \longrightarrow X \longrightarrow X^{\prime} \text { and } F^{\prime} \longrightarrow X^{\prime} \longrightarrow K V .
$$

By induction $X^{\prime}$ is sci, so that $X$ is sci as required. The codimension is obviously bounded below by $\operatorname{dim}\left(\mathbb{Q}\left(\pi_{o d d}(X)\right)\right.$, and we have described a procedure achieving this bound.

The following rearrangement result will be useful later.

Corollary 7.3. If $X$ occurs in a fibration

$$
X^{\prime} \longrightarrow X \longrightarrow K V
$$

with $X^{\prime}$ sci of s-codimension $c$, then $X$ is itself sci of s-codimension $c$.

Proof. By Theorem 7.1, $X^{\prime}$ has a model of the form $X^{\prime}=K V^{\prime} \rtimes F^{\prime}$ with $\pi_{*}\left(F^{\prime}\right)$ finite dimensional and in odd degrees and $X=K V \rtimes X^{\prime}$ with both $V$ and $V^{\prime}$ even and finite dimensional. By parity there can be no differential from $K V$ to $K V^{\prime}$, so

$$
X=K V^{\prime} \rtimes\left(K V \rtimes F^{\prime}\right) \simeq\left(K V^{\prime} \otimes K V\right) \rtimes F^{\prime},
$$

and we obtain a fibration

$$
F^{\prime} \longrightarrow X \longrightarrow K\left(V \oplus V^{\prime}\right) \text {. }
$$

By Theorem 7.1 again we deduce $X$ is sci of s-codimension $c$.

In terms of rational models we can restate the sci condition very simply. The result is immediate from Theorem 7.1 by taking a Sullivan model of the fibration.

Corollary 7.4. A space $X$ is sci if and only if $X$ has a cochain algebra model $(\Lambda V, d)$ where $d\left(V^{\text {even }}\right)=0$.

Finally, we relate the sci condition to our finiteness condition.

Lemma 7.5. Every sci space is strongly Noetherian.

Proof. First, by Theorem 7.1 and Lemma 4.7 every sci space has Noetherian cohomology. Second, by Corollary 7.4, if $R$ is a minimal Sullivan model for an sci space and $R^{\prime}$ is a sub-DGA of $R$ whose inclusion induces an injection on homotopy, then $R^{\prime}$ is a minimal Sullivan model for an sci space. 


\section{Examples}

It is quite easy to construct examples in rational homotopy theory, so we can see that various classes are distinct. This section on examples provides an interlude to the development; it illustrates the theory but does not play a part in the development.

\subsection{Homotopy invariant notions and cohomology rings}

We may impose a homotopy invariant condition on a space $X$ or a conventional condition on the cohomology ring $H^{*}(X)$. In the regular case there is no distinction by Theorem 4.3 , since rational graded connected commutative rings are regular if and only if they are polynomial on even degree generators.

In the ci case the homotopy invariant notion is strictly weaker than the notion for cohomology rings.

Proposition $8.1([16,4.2])$. If $H^{*}(X)$ is a complete intersection, then $X$ is formal, and there is a fibration

$$
S^{m_{1}} \times \cdots \times S^{m_{c}} \longrightarrow X \longrightarrow K V
$$

with $m_{1}, m_{2}, \ldots, m_{c}$ odd. In particular, $X$ is also sci.

Remark 8.2. By Theorem 7.1 a general sci space $X$ has a similar fibration with fibre an arbitrary space with finite dimensional odd homotopy. Those with $H^{*}(X)$ ci also have zero Postnikov invariants: there are vastly more sci spaces than those with ci cohomology. However Example 8.3 shows that even when the fibre is a product of odd spheres, the cohomology ring need not be ci.

Proof. We may suppose $H^{*}(X)=k\left[x_{1}, \ldots, x_{n}\right] /\left(f_{1}, \ldots, f_{c}\right)$ for suitable even degree generators $x_{1}, \ldots, x_{n}$ and regular sequence $f_{1}, \ldots, f_{c}$. Now let $V$ be a graded vector space with basis $x_{1}^{\prime}, \ldots, x_{n}^{\prime}$, where the degree of $x_{i}^{\prime}$ is the same as that of $x_{i}$, and let $W$ be a graded vector space with basis $\phi_{1}^{\prime}, \ldots, \phi_{c}^{\prime}$ where the codegree of $\phi_{i}^{\prime}$ is one less than that of $f_{i}$. We now take $X^{\prime}$ to have model $M\left(X^{\prime}\right)=\left(\Lambda(V) \rtimes \Lambda(W), d \phi_{1}^{\prime}=f_{1}^{\prime}, \ldots, d \phi_{c}^{\prime}=f_{c}^{\prime}\right)$. Since $f_{1}, \ldots, f_{c}$ is a regular sequence, $H^{*}\left(X^{\prime}\right) \cong H^{*}(X)$.

Now construct a map

$$
g: M\left(X^{\prime}\right) \longrightarrow C^{*}(X)
$$

by taking $g\left(x_{i}^{\prime}\right)$ to be a representative cycle for $x_{i} \in H^{*}(X)$. Since $f_{i}$ is trivial in $H^{*}(X)$, we may choose $\phi_{i} \in C^{*}(X)$ so that $d \phi_{i}=f_{i}$, and define $g\left(\phi_{i}^{\prime}\right)=\phi_{i}$. The resulting map $g$ is a cohomology isomorphism and therefore an equivalence.

The structure of $M\left(X^{\prime}\right)$ gives a fibration as claimed.

By contrast we may easily give an example of an sci space whose cohomology ring is not even Gorenstein.

Example 8.3. We take the rational space $X$ defined by the fibration

$$
S^{3} \times S^{3} \longrightarrow X \longrightarrow \mathbb{C} P^{\infty} \times \mathbb{C} P^{\infty}
$$

classified by the cohomology classes $u^{2}, u v \in H^{*}\left(\mathbb{C} P^{\infty} \times \mathbb{C} P^{\infty}\right)=\mathbb{Q}[u, v]$. It is not hard to calculate the cohomology of this space and see it is not Gorenstein.

On the other hand, $X$ is a finite Postnikov system and therefore Gorenstein in the sense of [10]. It is entertaining to make Gorenstein duality (the local cohomology spectral sequence) explicit in this case.

\subsection{Separating the hierarchy}

Since m-regular spaces are of the form $K V$, it is easy to see that there are sci spaces that are not regular. To give an example of an Gorenstein space that is not gci, we may use connected sums of manifolds, as in Example 8.4. Finally, there are many spaces with Noetherian cohomology that are not Gorenstein: two easy sources of examples are either finite dimensional spaces whose cohomology ring does not satisfy Poincaré duality, or Cohen-Macaulay rings which are not Gorenstein.

Example 8.4. We provide a space $X$ with $H^{*}(X)$ so that $X$ is Gorenstein but not gci. Almost any non-trivial connected sum of manifolds will do, but we give an explicit example.

First, note that if $M$ and $N$ are manifolds, their connected sum

$$
M \# N=\left(M^{\prime} \vee N^{\prime}\right) \cup e^{n}
$$

where $M^{\prime}$ is $M$ with a small disc removed, and similarly for $N^{\prime}$. By considering Lie models as in [11, 24.7], we obtain

$$
\pi_{*}(\Omega(M \# N))=\left(\pi_{*}\left(\Omega M^{\prime}\right) * \pi_{*}\left(\Omega N^{\prime}\right)\right) /(\alpha+\beta),
$$

where $*$ is the coproduct of graded Lie algebras, and where $\alpha$ and $\beta$ are the attaching maps for the top cells in $M$ and $N$.

Perhaps the simplest thing to try is $M=N=\mathbb{C} P^{2}$. Here we obtain

$$
\pi_{*}\left(\Omega\left(\mathbb{C} P^{2} \# \mathbb{C} P^{2}\right)\right)=\operatorname{Lie}\left(u_{1}, v_{1}\right) /\left(\left[u_{1}, u_{1}\right]+\left[v_{1}, v_{1}\right]\right),
$$


where $\operatorname{Lie}(V)$ denotes the free graded Lie algebra on $V$. This shows $\pi_{*}\left(\Omega\left(\mathbb{C} P^{2} \# \mathbb{C} P^{2}\right)\right)$ is finite, so $\mathbb{C} P^{2} \# \mathbb{C} P^{2}$ is gci, which also follows from the fact that its cohomology ring

$$
H^{*}\left(\mathbb{C} P^{2} \# \mathbb{C} P^{2}\right)=\mathbb{Q}\left[a_{2}, b_{2}\right] /\left(a^{2}-b^{2}, a b\right)
$$

is a complete intersection.

However, once we take three copies, we obtain

$$
H^{*}\left(\mathbb{C} P^{2} \# \mathbb{C} P^{2} \# \mathbb{C} P^{2}\right)=\mathbb{Q}\left[a_{2}, b_{2}, c_{2}\right] /\left(a^{2}=b^{2}=c^{2}, a b, a c, b c\right)
$$

with

$$
\pi_{*}\left(\Omega\left(\mathbb{C} P^{2} \# \mathbb{C} P^{2} \# \mathbb{C} P^{2}\right)\right)=\operatorname{Lie}\left(u_{1}, v_{1}, w_{1}\right) /\left(\left[u_{1}, u_{1}\right]+\left[v_{1}, v_{1}\right]+\left[w_{1}, w_{1}\right]\right),
$$

which is not finite, so that $\mathbb{C} P^{2} \# \mathbb{C} P^{2} \# \mathbb{C} P^{2}$ is not gci, giving the required example.

Example 8.5 ([11,12.(d) Example 1]). Here is an example of a space which is gci but not zci. Let $X$ be the space with model $R=\left(\Lambda\left(u_{3}, v_{3}, w_{5}\right), d w=u v\right)$. By the Eilenberg-Moore theorem $C_{*}(\Omega X) \simeq \operatorname{Hom}_{C^{*}(X)}(\mathbb{Q}, \mathbb{Q})$, so there is a map of graded rings $\Psi: Z \mathbf{D}(R) \rightarrow H_{*}(\Omega X)$ given by evaluating the endomorphism of the identity functor on $\mathbb{Q}$

$$
\Psi(\zeta)=\left(\zeta_{\mathbb{Q}}: \mathbb{Q} \rightarrow \Sigma_{n} \mathbb{Q}\right) \in H_{n}(\Omega X) .
$$

Clearly the image of $\Psi$ is contained in the centre of $H_{*}(\Omega X)$. The graded ring $H_{*}(\Omega X)$ is the enveloping algebra of the graded Lie algebra $L$, where $L$ is generated by three elements $U_{2}, V_{2}$ and $W_{4}$ and a single relation $[U, V]=W$. A straightforward calculation shows the centre of $H_{*}(\Omega X)$ to be the set $\left\{\mathbb{Q} W^{n} \mid n \geq 0\right\}$. Now suppose $X$ was zci, then we would have had appropriate elements $\zeta_{1}, \ldots, \zeta_{n} \in Z \mathbf{D}(R)$. Since the degree of $\zeta_{i}$ is non zero, $\Psi\left(\zeta_{i}\right)$ is either zero or $a_{i} W^{n_{i}}$ for some $n_{i}>0$.

Let $M$ be the $R$-module that is the cone of the map $\mathbb{Q} \stackrel{W}{\rightarrow} \Sigma_{4} \mathbb{Q}$. The module $M / \zeta_{1} / \cdots / \zeta_{n}$ must be a small $R$-module. Now consider the $C_{*}(\Omega X)$-module $\bar{M}=\operatorname{Hom}_{R}(\mathbb{Q}, M)$. By the Yoneda lemma, the map

$$
\bar{\zeta}_{i}=\operatorname{Ext}_{R}^{*}\left(\mathbb{Q}, \zeta_{i}\right): H_{*}(\bar{M}) \rightarrow H_{*+\left|\zeta_{i}\right|}(\bar{M})
$$

of $H_{*}(\Omega X)$-modules is simply multiplication by $\Psi\left(\zeta_{i}\right)$. It is easy to see that

$$
H_{*}(\bar{M})=H_{*}(\Omega X) /(W)=\mathbb{Q}[U, V]
$$

and therefore the induced map $\bar{\zeta}_{i}$ is zero on the homology of $\bar{M}$. An inductive argument reveals that $\operatorname{Hom}_{R}\left(\mathbb{Q}, M / \zeta_{1} / \cdots / \zeta_{n}\right)$ has infinitely many nonzero homology groups. However, since $M / \zeta_{1} / \cdots / \zeta_{n}$ is small and $C^{*}(X)$ is Gorenstein we see that the $C_{*}(\Omega X)$-module $\operatorname{Hom}_{R}\left(\mathbb{Q}, M / \zeta_{1} / \cdots / \zeta_{n}\right)$ has only finitely many nonzero homology groups, in contradiction.

The following example shows that the Noetherian condition is essential in the definition of gci.

Example 8.6 ([11, 12.(d) Example 3]). The space $X$ with model $\left(\Lambda\left(v_{2 a}, x_{2 b+1}, w_{2 a+2 b}\right), d w=v x\right)$ is not sci. For definiteness, we work with $a=b=1$, so that we have the model $\left(\Lambda\left(v_{2}, x_{3}, w_{4}\right), d w=v x\right)$.

Indeed, if $X$ is sci it must be in a fibration $S^{3} \longrightarrow X \longrightarrow K V$ where $V=\mathbb{Q}\{v, w\}$. But then in homotopy we have a short exact sequence

$$
0 \longrightarrow \pi_{*}\left(\Omega S^{3}\right) \longrightarrow \pi_{*}(\Omega X) \longrightarrow \pi_{*}(\Omega K V) \longrightarrow 0
$$

of graded Lie algebras, which implies that $\pi_{*}\left(\Omega S^{3}\right)$ is an ideal of $\pi_{*}(\Omega X)$. On the other hand, since $d w=v x$, the corresponding elements $\bar{v}_{1}, \bar{x}_{2}$ and $\bar{w}_{3}$ in the Lie algebra $\pi_{*}(\Omega X)$ satisfy $\bar{w}=[\bar{v}, \bar{x}]$, and we have a contradiction since $\pi_{*}\left(\Omega S^{3}\right)$ is generated by $\bar{x}$.

This is consistent with our general results since the cohomology ring is not Noetherian. Indeed, the cohomology ring $H^{*}(X)$ is $\mathbb{Q}[v]$ in even degrees, whilst all products of the odd degree elements $x, w x, w^{2} x, \ldots$ are zero.

Example 8.7. We are grateful to Don Stanley, for the following example which showed we needed to strengthen the hypotheses in an earlier version of Theorem 12.1.

We take $X$ to have model $\Lambda\left(a_{3}, b_{3}, c_{3}, e_{8}, f_{13}, g_{15}\right)$, where $a, b$ and $c$ are cycles and $d e=a b c, d f=a b e$ and $d g=e^{2}+2 c f$. This is evidently not sci (since de is non-zero). However, it has finite dimensional cohomology (for example by the Odd Spectral Sequence $[11,32.4]$, in which the only $d_{0}$ differential is $d_{0} g=e^{2}$ giving a finite dimensional $E_{1}$ term). This shows that gci does not imply sci without the additional hypothesis that $X$ is strongly Noetherian. To see explicitly that $X$ is not strongly Noetherian we refer to Example 13.10 below to see that the subalgebra generated by $a, b, c$ and $e$ is not Noetherian.

\section{Growth conditions}

In this section we prove perhaps the simplest implication between the ci conditions: for simply connected rational spaces of finite type, eci (and also zci) implies gci.

\subsection{Polynomial growth}

The rate of growth of homology groups is a natural homotopy invariant measurement of size. Similarly, the rate of growth of resolutions is a natural measure of complexity. 
We will be working over $H^{*}(X)$, so it is natural to assume that our modules $M$ are locally finite in the sense that $H^{*}(M)$ is cohomologically bounded below and $\operatorname{dim}_{\mathbb{Q}}\left(H^{i}(M)\right)$ is finite for all $i$.

Definition 9.1. (i) We say that a locally finite module $M$ has polynomial growth of degree $d$, and write growth(M) $\leq d$, if there is a polynomial $p(x)$ of degree $d$ with

$$
\operatorname{dim}_{\mathbb{Q}}\left(H^{n}(M)\right) \leq p(n)
$$

for all $n>>0$.

(ii) An $R$ module $M$ has complexity $\leq n$ if $\operatorname{Ext}_{R}^{*}(M, k)$ has polynomial growth of degree $n-1$.

Remark 9.2. (i) Note that a complex with bounded homology has growth $\leq-1$. For complexes which do not have bounded homology, by adding a constant to the polynomial, we may insist that the bound applies for all $n \geq 0$.

(ii) When $R$ is an ungraded local ring and $M$ is concentrated in degree 0 , the complexity refers directly to the rate of growth of a minimal projective resolution of $M$.

(iii) For a ci local ring, the codimension is the complexity of the residue field. When $R=C^{*}(X)$

$$
\operatorname{cX}_{C^{*}(X)}(\mathbb{Q})=\operatorname{growth}\left(H^{*}(\Omega X)\right)+1,
$$

and the complexity of $\mathbb{Q}$ is the g-codimension when $X$ is gci.

\subsection{Mapping cones reduce degree by one}

We use the following estimate on growth.

Lemma 9.3. Given cohomologically bounded below locally finite modules $M$ and $N$ in a triangle

$$
\Sigma_{n} M \stackrel{\chi}{\longrightarrow} M \longrightarrow N
$$

with $n \neq 0$, then

$$
\operatorname{growth}(M) \leq \operatorname{growth}(N)+1 \text {. }
$$

Proof. The homology long exact sequence of the triangle includes

$$
\cdots \longrightarrow H^{i-n}(M) \stackrel{\chi}{\longrightarrow} H^{i}(M) \longrightarrow H^{i}(N) \longrightarrow \cdots .
$$

This shows

$$
\operatorname{dim}_{\mathbb{Q}}\left(H^{i}(M)\right) \leq \operatorname{dim}_{\mathbb{Q}}\left(H^{i}(N)\right)+\operatorname{dim}_{\mathbb{Q}}\left(\chi H^{i-n}(M)\right) .
$$

Iterating $s$ times, we find

$$
\operatorname{dim}_{\mathbb{Q}}\left(H^{i}(M)\right) \leq \operatorname{dim}_{\mathbb{Q}}\left(H^{i}(N)\right)+\operatorname{dim}_{\mathbb{Q}}\left(\chi H^{i-n}(N)\right)+\cdots+\operatorname{dim}_{\mathbb{Q}}\left(\chi^{s-1} H^{i-(s-1) n}(N)\right)+\operatorname{dim}_{\mathbb{Q}}\left(\chi^{s} H^{i-s n}(M)\right) .
$$

To obtain growth estimates, it is convenient to collect the dimensions of the homogeneous parts into the Hilbert series $h_{M}(t)=\sum_{n} \operatorname{dim}_{\mathbb{Q}}\left(H^{i}(M)\right) t^{i}$. An inequality between such formal series means that it holds between all coefficients.

First suppose that $n>0$. Since $H^{*}(M)$ is bounded below, if $h_{M}(t)$ is the Hilbert series of $H^{*}(M)$ then we have

$$
h_{M}(t) \leq h_{N}(t)\left(1+t^{n}+t^{2 n}+\cdots\right)=\frac{h_{N}(t)}{1-t^{n}},
$$

giving the required growth estimate.

If $n=-n^{\prime}<0$ we rearrange to obtain

$$
N^{\prime} \longrightarrow M \longrightarrow \Sigma_{n^{\prime}} M
$$

where $N^{\prime}=\Sigma_{n^{\prime}-1} N$ and argue in the same way.

\subsection{Growth of eci spaces}

The implication we require is now straightforward.

Theorem 9.4. If $X$ is eci then it is also gci, and if $X$ has e-codimension $c$ it has g-codimension $\leq c$.

Proof. It is sufficient to show $C^{*}(\Omega X) \simeq \mathbb{Q} \otimes_{C^{*}(X)} \mathbb{Q}$ has polynomial growth.

By hypothesis there is an appropriate regular space $K$ and self maps

$$
\gamma_{1}: M_{0} \rightarrow \Sigma_{\left|\gamma_{1}\right|} M_{0}, \gamma_{2}: M_{1} \rightarrow \Sigma_{\left|\gamma_{1}\right|} M_{1}, \ldots, \gamma_{c}: M_{c-1} \rightarrow \Sigma_{\left|\gamma_{c}\right|} M_{c-1}
$$

of non-zero degree in $\mathbf{D}\left(C^{*}\left(X \times_{K} X\right)\right)$, so that $M_{i}$ is the cone of $\gamma_{i}$ and $M_{c}$, which is the cone of $\gamma_{c}$, is small. Thus, applying $\mathbb{Q} \otimes_{C^{*}(X)}(\cdot)$ to $M_{c}$ we obtain a complex with growth $\leq-1$. By the lemma if we apply $\mathbb{Q} \otimes_{C^{*}(X)}(\cdot)$ to $M_{c-1}$ we obtain a complex of growth $\leq 0$. Doing this repeatedly, we deduce that when we apply $\mathbb{Q} \otimes_{C^{*}(X)}(\cdot)$ to $\mathbb{Q}$ itself we obtain a complex with growth $\leq c-1$ as required. 
The proof above, with minor changes, also yields the following theorem.

Theorem 9.5. If $X$ is zci then it is also gci, and if $X$ has $z$-codimension $c$ it has $g$-codimension $\leq c$.

\section{Sci spaces are eci spaces}

In this section we show that sci spaces (defined by a particular construction) have a periodic module theory in the sense that they are eci. This may not be too surprising, but the particular way in which bimodules and fibrations are used is of some interest.

Theorem 10.1. If $X$ is an sci space of $s$-codimension $c$, then it is eci of e-codimension $c$.

Remark 10.2. The construction will show that all the maps building the small bimodule are of positive degree, so that Lemma 5.1 shows that if $X$ is sci then all $C^{*}(X)$-modules in $\mathcal{F} G$ are virtually small. In fact, the small bimodule we build is $C^{*}\left(X \times_{K V} X\right)$, for an appropriate choice of $K V$.

We will upgrade the conclusion to show that if $X$ is a pure sci space then $X$ is zci in Section 11 .

\subsection{Fibration lemmas}

We will repeatedly use two elementary lemmas. The first is very well known.

\section{Lemma 10.3. If}

$$
F \longrightarrow E \stackrel{p}{\longrightarrow} B
$$

is a fibration with a section s, then there is a fibration

$$
\Omega F \longrightarrow B \stackrel{s}{\longrightarrow} E .
$$

Proof. We start from the square

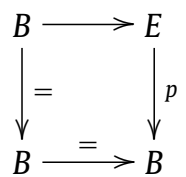

and take iterated fibres.

The second lemma is a Third Isomorphism Theorem for fibrations.

Lemma 10.4. Given fibrations $Y \longrightarrow B \longrightarrow C$, if $F=$ fibre $(B \longrightarrow C)$ there is a fibration

$$
\Omega F \longrightarrow Y \times_{B} Y \longrightarrow Y \times_{C} Y .
$$

Proof. We start from the cube

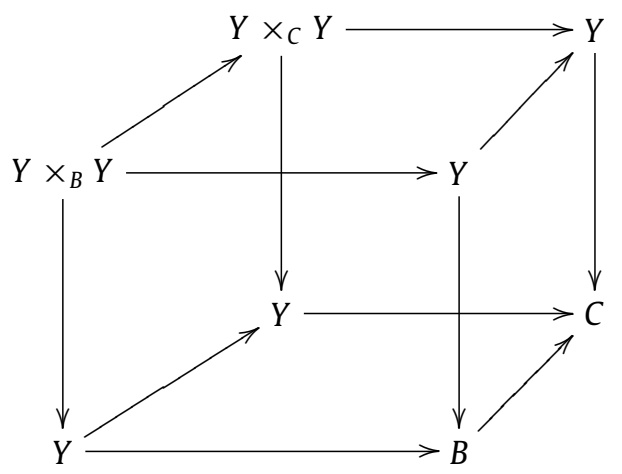

and take iterated fibres.

\subsection{Building bimodules}

It is worth isolating the process that we use repeatedly to build bimodules. Abstracted from its context, the proof is extremely simple. The strength of the result is that the cofibre sequence is one of $C^{*}(Y)$-modules. 
Proposition 10.5. Suppose given a fibration

$$
\Omega S^{m} \longrightarrow X \stackrel{f}{\longrightarrow} Y .
$$

(i) If $m$ is odd, then there is a cofibre sequence of $C^{*}(Y)$-modules

$$
\Sigma_{m-1} C^{*}(X) \longleftarrow C^{*}(X) \stackrel{f^{*}}{\longleftarrow} C^{*}(Y) .
$$

(ii) If $m$ is even, then there is a cofibre sequence of $C^{*}(Y)$-modules

$$
\Sigma_{2 m-2} C^{*}(X) \longleftarrow C^{*}(X) \longleftarrow F
$$

with $F$ small. More precisely, $F$ is built from two copies of $C^{*}(Y)$ in the sense that there is a cofibre sequence

$$
\Sigma_{m-1} C^{*}(Y) \longleftarrow F \longleftarrow C^{*}(Y)
$$

of $C^{*}(Y)$-modules.

Proof. We take a relative Sullivan model for $f^{*}$. This has the form $C^{*}(Y) \rtimes C^{*}\left(\Omega S^{m}\right)$.

If $m$ is odd, the relative Sullivan model is of the form $C^{*}(X) \simeq C^{*}(Y) \rtimes \Lambda\left(z_{m-1}\right)$, where $z$ is a polynomial generator. The quotient of this by the DG- $C^{*}(Y)$-submodule $C^{*}(Y) \cdot z^{0}$ is again a $C^{*}(Y)$-module, and the composite

$$
\Sigma_{m-1} C^{*}(X) \stackrel{z}{\longrightarrow} C^{*}(X) \longrightarrow C^{*}(X) / C^{*}(Y)
$$

is an isomorphism as required.

If $m$ is even, the relative Sullivan model is of the form

$$
C^{*}(X) \simeq C^{*}(Y) \rtimes \Lambda\left(z_{m-1}, t_{2 m-2}\right) .
$$

This time $t$ is a polynomial generator, and $z$ is an exterior generator. Accordingly we let $F$ be the DG- $C^{*}(Y)$-submodule generated by $t^{0}$ and $z$, so that $C^{*}(X) / F$ is again a $C^{*}(Y)$-module. It is isomorphic to $\Sigma_{2 m-2} C^{*}(X)$ in the sense that the composite

$$
\Sigma_{2 m-2} C^{*}(X) \stackrel{t}{\longrightarrow} C^{*}(X) \longrightarrow C^{*}(X) / F
$$

is an isomorphism.

Remark 10.6. As an example, we observe that this shows that an sci space of s-codimension 1 is a zci space of z-codimension 1. Indeed, by hypothesis, we have a fibration $S^{m} \longrightarrow X \longrightarrow K V$, and hence by pullback a fibration

$$
S^{m} \longrightarrow X \times_{K V} X \longrightarrow X
$$

with a section $\Delta: X \longrightarrow X \times_{K V} X$. By Lemma 10.3, we obtain a fibration

$$
\Omega S^{m} \longrightarrow X \stackrel{\Delta}{\longrightarrow} X \times_{K V} X
$$

so we may apply Proposition 10.5 with $Y=X \times_{K V} X$, noting that a $C^{*}(Y)$-module is then a $C^{*}(X)$-bimodule. If $m$ is odd we then get a cofibre sequence

$$
\Sigma_{m-1} C^{*}(X) \stackrel{\tilde{x}}{\longleftarrow} C^{*}(X) \longleftarrow C^{*}\left(X \times_{K V} X\right)
$$

of bimodules. As in Section 5.3 note that $\tilde{\chi}$ gives an element of $Z \mathbf{D}\left(C^{*}(X)\right)$ by tensoring down, in the sense that for any $C^{*}(X)$-module $M$ we apply $M \otimes_{C^{*}(X)}(\cdot)$ to get a cofibre sequence

$$
\Sigma_{m-1} M \stackrel{\chi}{\longleftarrow} M \longleftarrow C^{*}(X) \otimes_{C^{*}(K V)} M .
$$

If $M$ is finitely generated, then it is small as a $C^{*}(K V)$-module by Proposition 4.4 showing that the fibre of $\chi$ is small as required.

The argument when $m$ is even is precisely similar.

\subsection{The proof}

We now have the necessary ingredients for proving Theorem 10.1 .

We suppose $X$ is sci of s-codimension $c$, so that we may form $X=X_{c}$ in $c$ steps from $X_{0}=K V$ using fibrations

$$
S^{n_{i}} \longrightarrow X_{i} \longrightarrow X_{i-1} \text {. }
$$

It will simplify the argument to assume all the spheres are odd dimensional, as we may do by Lemma 7.2. 
We must show that $C^{*}(X)$ builds $C^{*}\left(X \times_{K V} X\right)$ as a bimodule (i.e., as a $C^{*}\left(X \times_{K V} X\right)$-module) using $s$ cofibre sequences. It is convenient to write $X_{i}^{e}=X \times_{X_{i}} X$, and $X^{e}=X_{0}^{e}$, so that we want to work with $C^{*}\left(X^{e}\right)$-modules. However, since we have maps

$$
X=X_{s} \longrightarrow X_{s-1} \longrightarrow \cdots \longrightarrow X_{0}=K V,
$$

we have maps

$$
X_{s}=X_{s}^{e} \longrightarrow X_{s-1}^{e} \longrightarrow \cdots \longrightarrow X_{0}^{e}=X^{e},
$$

so we may view $C^{*}\left(X_{i}^{e}\right)$-modules as $C^{*}\left(X_{0}^{e}\right)$-modules by restriction.

We are ready to apply our fibration lemmas.

Pulling back the fibration along $X_{i} \longrightarrow X_{i-1}$ we obtain a fibration

$$
S^{n_{i}} \longrightarrow X_{i} \times_{X_{i-1}} X_{i} \stackrel{\pi_{1}}{\longrightarrow} X_{i}
$$

with a section given by the diagonal $\Delta$. Applying Lemma 10.3 we obtain a fibration

$$
\Omega S^{n_{i}} \longrightarrow X_{i} \stackrel{\Delta}{\longrightarrow} X_{i} \times_{X_{i-1}} X_{i} .
$$

Similarly, applying Lemma 10.4 to $X_{s} \longrightarrow X_{i} \longrightarrow X_{i-1}$ where $s \geq i$, we obtain a fibration

$$
\Omega S^{n_{i}} \longrightarrow X_{i}^{e} \longrightarrow X_{i-1}^{e} \text {. }
$$

Now using the first of these, Proposition 10.5 gives a cofibration

$$
\Sigma_{n_{s}} C^{*}(X) \longleftarrow C^{*}(X) \longleftarrow C^{*}\left(X_{s-1}^{e}\right),
$$

of $C^{*}\left(X_{s-1}^{e}\right)$-modules, which we view as a cofibration of $C^{*}\left(X^{e}\right)$-modules by pullback. Successive fibrations give

$$
\Sigma_{n_{i}} C^{*}\left(X_{i}^{e}\right) \longleftarrow C^{*}\left(X_{i}^{e}\right) \longleftarrow C^{*}\left(X_{i-1}^{e}\right),
$$

until we reach

$$
\Sigma_{n_{1}} C^{*}\left(X_{1}^{e}\right) \longleftarrow C^{*}\left(X_{1}^{e}\right) \longleftarrow C^{*}\left(X^{e}\right),
$$

so that

$$
C^{*}(X)=C^{*}\left(X_{s}^{e}\right)=C^{*}\left(X_{0}^{e}\right)=C^{*}\left(X^{e}\right)
$$

as required.

This shows that an sci space of s-codimension $c$ is eci of e-codimension no greater than $c$. But since an sci space of scodimension $c$ is gci of g-codimension $c$, Theorem 9.4 shows that its e-codimension cannot be smaller than $c$.

\section{Hochschild cohomology and pure Sullivan algebras}

In this section we lift several constructions to the level of bimodules when the space $X$ is pure. To start with, we upgrade the conclusion of Section 10 for pure spaces to give the required conclusion that a pure sci space is also zci.

Theorem 11.1. If $X$ is a pure sci space of s-codimension c, then it is zci of $z$-codimension $c$.

Building on this result, we turn to Hochschild cohomology in Section 11.5.

\subsection{Upgrading bimodule maps}

In view of Theorem 10.1 and Remark 10.2 to prove Theorem 11.1 we need to show that the maps of bimodules used in the constructions of Section 10 all lift to elements of $Z \mathbf{D}(R)$.

Recall that we have maps

$$
X=X_{s} \longrightarrow X_{s-1} \longrightarrow \cdots \longrightarrow X_{0}=K V,
$$

such that the homotopy fibre of each map is an odd sphere and each map induces a surjection on homotopy groups. Concentrating on a single map in this sequence we may assume that there is a fibration sequence $S^{2 n+1} \longrightarrow Y \longrightarrow Z$, and a map $X \longrightarrow Y$ such that

(i) $\pi_{*}(Y) \longrightarrow \pi_{*}(Z)$ is surjective,

(ii) $\pi_{*}(X) \longrightarrow \pi_{*}(Y)$ is surjective, with kernel concentrated in odd degrees, and

(iii) $\pi_{*}(X)$ is finite dimensional and $X$ is a pure space. 
We write $A=C^{*}(X), B=C^{*}(Y)$ and $C=C^{*}(Z)$. It is shown in Section 10 (see Lemma 10.4 and Proposition 10.5 ) that there is a cofibre sequence of $A \otimes_{C} A$-modules

$$
A \otimes_{C} A \rightarrow A \otimes_{B} A \stackrel{\varphi}{\rightarrow} \Sigma_{2 n} A \otimes_{B} A .
$$

To obtain an element of $Z \mathbf{D}(A)$ we proceed as follows. For each $A$-module $M$, we apply $-\otimes_{A} M$ to obtain the cofibre sequence

$$
A \otimes_{C} M \rightarrow A \otimes_{B} M \stackrel{\varphi \otimes_{A} M}{\longrightarrow} \Sigma_{2 n} A \otimes_{B} M .
$$

To establish the zci condition, we must check it is natural for maps of $A$-modules.

Proposition 11.2. There is a morphism $\zeta: A \rightarrow \Sigma_{2 n} A$ of $A \otimes_{C} A$-modules (i.e., an element $\zeta \in H H^{2 n}(A \mid C)$ ) such that

$\zeta \otimes_{B} A \simeq \varphi$.

We will turn to the proof shortly (and complete it in Section 11.4), but for the present we just observe that it has the desired consequence.

Corollary 11.3. There is a natural transformation $z$ of the identity functor on A-modules such that for every A-module $M$

$$
z\left(A \otimes_{B} M\right) \simeq \varphi \otimes_{A} M .
$$

Proof. The natural transformation $z$ that $\zeta$ induces on $A$-modules is given by $z(M)=\zeta \otimes_{A} M$. We easily verify this has the required property:

$$
\begin{aligned}
z\left(A \otimes_{B} M\right) & =\zeta \otimes_{A}\left(A \otimes_{B} M\right) \\
& =\zeta \otimes_{B} M \\
& =\left(\zeta \otimes_{B} A\right) \otimes_{A} M \\
& =\varphi \otimes_{B} M .
\end{aligned}
$$

This completes the proof of Theorem 11.1 modulo the proof of Proposition 11.2.

\subsection{Models for spaces}

In this subsection we introduce the concrete models we need for the proof of Proposition 11.2. We take specific minimal Sullivan models $A, B$, and $C$ for $X, Y$ and $Z$ respectively as follows.

(1) $C=(\Lambda W, d)$ with $W$ finite dimensional.

(2) $B=\left(\Lambda\left(W \oplus \mathbb{Q} x_{2 n+1}\right), d\right)$, containing $C$ as a sub-algebra. We denote $W \oplus \mathbb{Q} x$ by $V$.

(3) $A=(\Lambda(V \oplus U), d)$, containing $B$ as a sub-algebra, with $U$ concentrated in odd degrees and finite dimensional. We also assume that $d(U) \subset \Lambda W$, this is possible because $X$ has a pure Sullivan model.

Let $X_{Y}^{e}=X \times_{Y} X$ and let $X_{Z}^{e}=X \times_{Z} X$. The cochain algebras $A_{B}^{e}=A \otimes_{B} A$ and $A_{C}^{e}=A \otimes_{C} A$ are minimal Sullivan models for $X_{Y}^{e}$ and $X_{Z}^{e}$. We can write these cochain algebras explicitly as well.

- $A_{B}^{e}=\left(\Lambda\left(V \oplus U_{l} \oplus U_{r}\right), d\right)$ where $U_{l}=\left\{u_{l} \mid u \in U\right\}$ and $U_{r}=\left\{u_{r} \mid u \in U\right\}$. The differential $d$ is the obvious one satisfying $d\left(u_{l}\right)=d\left(u_{r}\right)=d(u) \in \Lambda W$ and so $u_{l}-u_{r}$ is always a cocycle.

- $A_{C}^{e}=\left(\Lambda\left(W \oplus \mathbb{Q}\left\{x_{l}, x_{r}\right\} \oplus U_{l} \oplus U_{r}\right), d\right)$.

Remark 11.4. There are three important morphisms for $X_{Y}^{e}$ :

(1) $l: X_{Y}^{e} \rightarrow X$ which is mapping to the left component,

(2) $r: X_{Y}^{e} \rightarrow X$ which is mapping to the right component and

(3) $\Delta: X \rightarrow X_{Y}^{e}$ which is the diagonal.

The algebraic counterparts of these maps are $l: A \rightarrow A_{B}^{e}, r: A \rightarrow A_{B}^{e}$ and $\Delta: A_{B}^{e} \rightarrow A$. The morphism $l$ is the map to the left component of $A \otimes_{B} A=A_{B}^{e}$, it is defined by $l(u)=u_{l}$ for $u \in U$ and $l(v)=v$ for $v \in V$. The description of $r: A \rightarrow A_{B}^{e}$ is precisely similar. The diagonal map $\Delta: A_{B}^{e} \rightarrow A$ is defined by $\Delta\left(u_{l}\right)=\Delta\left(u_{r}\right)=u$ and $\Delta$ is the identity on $\mathbb{Q} x$ and $W$. There are similar maps for $X_{Z}^{e}$ and $A_{C}^{e}$. Note that $A$ is a $A_{C}^{e}$-module via the morphism $\Delta: A_{C}^{e} \longrightarrow A$.

\subsection{Some useful fibrations}

In the next few subsections we use the concrete models of Section 11.2 to study the homotopy theory of the spaces $X, Y$ and $Z$ needed for the proof of Proposition 11.2.

The following fibrations will be central to the proof.

Lemma 11.5. There are the following fibration sequences,

(1) $X_{Y}^{e} \stackrel{\Delta}{\rightarrow} X_{Z}^{e} \rightarrow S(\mathbb{Q} x)$;

(2) $X \stackrel{\Delta}{\rightarrow} X_{Y}^{e} \rightarrow S(U)$;

(3) $X \stackrel{\Delta}{\rightarrow} X_{Z}^{e} \rightarrow S(U \oplus \mathbb{Q} x)$;

where the bases are products of odd spheres with the indicated homotopy groups. 
Proof. We reformulate the lemma algebraically: there are the following cofibration sequences of Sullivan algebras.

(1) $L_{1}=\left(\Lambda \mathbb{Q} x^{\prime}, 0\right) \rightarrow A_{C}^{e} \stackrel{\Delta}{\rightarrow} A_{B}^{e}$, where $x^{\prime} \mapsto x_{l}-x_{r}$.

(2) $L_{2}=(\Lambda U, 0) \rightarrow A_{B}^{e} \stackrel{\Delta}{\rightarrow} A$, where $u \mapsto u_{l}-u_{r}$.

(3) $L_{1} \otimes_{\mathbb{Q}} L_{2}=\left(\Lambda\left(\mathbb{Q} x^{\prime} \oplus U\right), 0\right) \rightarrow A_{C}^{e} \stackrel{\Delta}{\rightarrow} A$.

Since the composite $\left(\Lambda \mathbb{Q} x^{\prime}, 0\right) \rightarrow A_{C}^{e} \stackrel{\Delta}{\rightarrow} A_{B}^{e}$ is the trivial morphism, there is a natural morphism $\epsilon: A_{C}^{e} \otimes_{L_{1}} \mathbb{Q} \rightarrow A_{B}^{e}$. It is easy to see that $\epsilon$ is an isomorphism on homotopy groups. The proof for the two other cofibration sequences is similar.

We shall make two uses of these fibrations. The first use is to build relative cofibrant models for our cochain algebras. An $A_{C}^{e}$-cofibrant model for $A_{B}^{e}$ is

$$
\tilde{A_{B}^{e}}=\left(\Lambda\left(W \oplus \mathbb{Q}\left\{x_{l}, x_{r}, z_{2 n}\right\} \oplus U_{l} \oplus U_{r}\right), d\right) \quad \text { where } d z=x_{l}-x_{r} .
$$

It is easy to see that the obvious morphism $\tilde{A}_{B}^{e} \rightarrow A_{B}^{e}$ is indeed a weak equivalence. Similarly a cofibrant model for $A$ over $\tilde{A_{B}^{e}}$ (and therefore also over $A_{C}^{e}$ ) is given by the formula

$$
\tilde{A}=\left(\Lambda\left(W \oplus \mathbb{Q}\left\{x_{l}, x_{r}, z_{2 n}\right\} \oplus U_{l} \oplus U_{r} \oplus \tilde{U}\right), d\right) \quad \text { where } \tilde{U}=\Sigma_{1} U \quad \text { and } \quad d(\tilde{u})=u_{l}-u_{r} .
$$

The second use of Lemma 11.5 is in defining a strange and useful space $T$.

Lemma 11.6. Let $T$ be the homotopy fibre of the $\operatorname{map} X_{Z}^{e} \rightarrow S(U)$.

(1) There is a fibration sequence $X \rightarrow T \rightarrow S(\mathbb{Q} x)$.

(2) The following is a homotopy pullback square

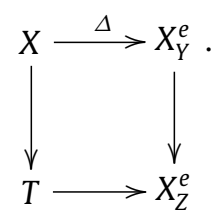

Proof. An $A_{C}^{e}$-cofibrant cochain model for $T$ is given by the formula

$$
F=\left(\Lambda\left(W \oplus Q\left\{x_{l}, x_{r}\right\} \oplus U_{l} \oplus U_{r} \oplus \tilde{U}\right), d\right) \quad \text { where } \tilde{U}=\Sigma_{1} U \text { and } \quad d(\tilde{u})=u_{l}-u_{r} .
$$

Note that $u_{l}-u_{r}$ is a cocycle because $d(U) \subset \Lambda W$. From this model the fibration sequence $X \rightarrow T \rightarrow S(\mathbb{Q} x)$ is evident.

Let $X^{\prime}$ be the homotopy pullback of the diagram:

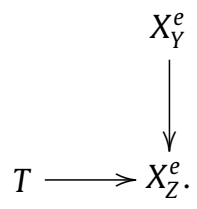

A cochain algebra model for $X^{\prime}$ is $F \otimes_{A_{C}^{e}} \tilde{A}_{B}^{e}=\left(\Lambda\left(W \oplus Q\left\{x_{l}, x_{r}\right\} \oplus U_{l} \oplus U_{r} \oplus \tilde{U} \oplus \mathbb{Q} z_{2 n}\right), d\right)$ which is clearly isomorphic to $\tilde{A}$. Moreover, the morphism $\tilde{A}_{B}^{e} \rightarrow F \otimes_{A_{C}^{e}} \tilde{A}_{B}^{e} \cong \tilde{A}$ is indeed the diagonal $\Delta$.

\subsection{Lifting the map of bimodules}

We can now prove Proposition 11.2. Recall the cochain model $F$ for $T$ given in the proof of Lemma 11.6. The fibration $X \rightarrow T \rightarrow S(\mathbb{Q} x)$ induces an exact sequence of $F$-modules (and therefore also of $A_{C}^{e}$-modules):

$$
F \rightarrow \tilde{A} \stackrel{\zeta}{\rightarrow} \Sigma_{2 n} \tilde{A} .
$$

We will require an explicit description of $\zeta$. It is defined by

- $\zeta\left(f z^{q}\right)=f z^{q-1}$ and

- $\zeta(f)=0$ if $f$ is not divisible by $z$.

Similarly, the fibration sequence $X_{Y}^{e} \rightarrow X_{Z}^{e} \rightarrow S(\mathbb{Q} x)$ gives rise to an exact sequence

$$
A_{C}^{e} \rightarrow \tilde{A_{B}^{e}} \stackrel{\varphi}{\rightarrow} \Sigma_{2 n} \tilde{A_{B}^{e}}
$$

of $A_{C}^{e}$-modules. An explicit description of $\varphi$ is given by

- $\varphi\left(f z^{q}\right)=f z^{q-1}$ and

- $\varphi(f)=0$ if $f$ is not divisible by $z$. 
As a cofibrant replacement of $B$ over $B \otimes_{\mathcal{C}} B$, we take $\tilde{B}=\left(\Lambda\left(W \oplus \mathbb{Q}\left\{x_{l}, x_{r}, z_{2 n}\right\}\right), d\right)$ with $d z=x_{l}-x_{r}$. We can now prove the following proposition, which is an explicit cochain level version of Proposition 11.2.

Proposition 11.7. There is a natural equivalence

$$
\varphi \simeq \zeta \otimes_{\tilde{B}} \tilde{A} .
$$

Proof. We shall define a cochain algebra model $\hat{A}$ for $X$, which is cofibrant over $\tilde{B}$. Let $\hat{A}=\left(\Lambda\left(W \oplus \mathbb{Q}\left\{x_{l}, x_{r}, z_{2 n}\right\} \oplus U_{l}\right), d\right)$ where $d z=x_{l}-x_{r}$. The morphism $\zeta$ is equivalent to the obvious morphism $\hat{\zeta}: \hat{A} \rightarrow \Sigma_{2 n} \hat{A}$. It is now easy to see there is an equality of morphisms of $A_{C}^{e}$-modules:

$$
\varphi=\hat{\zeta} \otimes_{\tilde{B}} \hat{A} .
$$

Remark 11.8. Proposition 10.5 shows that the fibration $S^{2 n+1} \rightarrow Y \rightarrow Z$ yields an exact sequence:

$$
B \otimes_{C} B \rightarrow \tilde{B} \stackrel{\psi}{\rightarrow} \Sigma_{2 n} \tilde{B} .
$$

It is easy to see that $\varphi=A \otimes_{B} \psi \otimes_{B} A$ (note that $\psi$ is a morphism of $B \otimes_{C} B$-modules, which justifies tensoring over $B$ on the left and right).

\subsection{Hochschild cohomology}

Pushing the ideas of this section a little further, we may make calculations of Hochschild cohomology. To start with, we adapt the algebraic notation for Hochschild cohomology to cochain algebras.

Notation 11.9. If $X \rightarrow Y$ is a fibration of spaces, set

$$
H H^{*}(X \mid Y):=\operatorname{Ext}_{C^{*}(X \times Y X)}^{*}\left(C^{*}(X), C^{*}(X)\right) \text {. }
$$

Note that we consider $C^{*}(X)$ as a $C^{*}\left(X \times_{Y} X\right)$-module via the diagonal map $X \rightarrow X \times_{Y} X$.

We will be applying this to sci spaces, and use the notation of Section 10:X $=X_{S}$ is an sci space of s-codimension $s$, so that for $1 \leq i \leq s$ we have fibrations $S^{n_{i}} \rightarrow X_{i} \rightarrow X_{i-1}$ with $n_{i}$ odd and $X_{0}=K V$, where $V$ is finite dimensional and even.

Theorem 11.10. If $X$ is a pure sci space as above, the Hochschild cohomology is given by

$$
H H^{*}(X \mid K V)=H^{*}(X)\left[\left[\zeta_{1}\right]\right] \ldots\left[\left[\zeta_{s}\right]\right],
$$

where the degree of $\zeta_{i}$ is $n_{i}-1$.

Remark 11.11. (i) The formula is to be interpreted as obtained by adjoining power series variables $\zeta_{i}$ in succession. More precisely, one forms the polynomial ring and completes with respect to the homogeneous ideals generated by powers of the variable. In particular, if $X$ is finite dimensional the ring is a polynomial ring.

(ii) One might hope to apply Theorem 11.10 to upgrade the construction of Section 10 to show $X$ is zci. This is not true in general, but on the other hand, the Hochschild cohomology calculation is obtained by upgrading the construction.

The theorem evidently follows by repeated application of the following general result about fibrations with fibre an odd sphere.

Proposition 11.12. Suppose given a fibration sequence $S^{2 n+1} \longrightarrow Y \longrightarrow Z$, and a fibration $X \longrightarrow Y$. Assuming (i) $\pi_{*}(Y) \longrightarrow$ $\pi_{*}(Z)$ is surjective, (ii) $\pi_{*}(X) \longrightarrow \pi_{*}(Y)$ is surjective, with kernel concentrated in odd degrees, and (iii) $\pi_{*}(X)$ is finite dimensional and $X$ is a pure space, we have

$$
H H^{*}(X \mid Z) \cong H H^{*}(X \mid Y)[[\zeta]],
$$

where $\zeta$ is of degree $2 n$.

This will be proved in Section 11.6 below.

\subsection{Proof of Proposition 11.12}

Using our cofibrant models, we have explicit complexes for calculating Hochschild cohomology:

$$
H H^{*}(A \mid C)=H^{*}\left(\operatorname{End}_{A_{C}^{e}}(\tilde{A})\right) \text { and } H H^{*}(A \mid B)=H^{*}\left(\operatorname{End}_{\tilde{A}_{B}^{e}}(\tilde{A})\right) \text {. }
$$

In these terms, we may state Proposition 11.12 more explicitly as follows.

\section{Proposition 11.13.}

$$
H^{*}\left(\operatorname{End}_{A_{C}^{e}}(\tilde{A})\right)=H^{*}\left(\operatorname{End}_{\hat{A}_{B}^{e}}(\tilde{A})\right)[[\zeta]]
$$

Proof. Let $R=H^{*}\left(\operatorname{End}_{A_{C}^{e}}(\tilde{A})\right)$ and let $Q=H^{*}\left(\operatorname{End}_{\tilde{A}_{B}^{e}}(\tilde{A})\right)$. Note that both $R$ and $Q$ are graded-commutative, because Hochschild cohomology is always graded-commutative. To prove the proposition we need several ingredients. The first 
ingredient is a short exact sequence

$$
\Sigma^{2 n} R \stackrel{\zeta}{\rightarrow} R \rightarrow Q
$$

of $R$-modules.

Consider the morphism $F \stackrel{p}{\rightarrow} \tilde{A}$ of Lemma 11.6. Applying the functor $\operatorname{Hom}_{A_{C}^{e}}(-, \tilde{A})$ yields a morphism $\operatorname{End}_{A_{C}^{e}}(\tilde{A}) \stackrel{p^{*}}{\rightarrow}$ $\operatorname{Hom}_{A_{C}^{e}}(F, \tilde{A})$. Since $\tilde{A}$ is a $\tilde{A_{B}^{e}}$-module there is an adjunction:

$$
\operatorname{Hom}_{A_{C}^{e}}(F, \tilde{A}) \cong \operatorname{Hom}_{\tilde{A}_{B}^{e}}\left(\tilde{A_{B}^{e}} \otimes_{A_{C}^{e}} F, \tilde{A}\right) \cong \operatorname{End}_{\hat{A}_{B}^{e}}(\tilde{A})
$$

(the isomorphism $\tilde{A_{B}^{e}} \otimes_{A_{C}^{e}} F \cong \tilde{A}$ is Part 2 of Lemma 11.6). Thus $p^{*}$ is a map $\operatorname{End}_{A_{C}^{e}}(\tilde{A}) \rightarrow \operatorname{End}_{\tilde{A}_{B}^{e}}(\tilde{A})$. On the other hand, we have the natural multiplicative change of rings map $\iota: \operatorname{End}_{\tilde{A}_{B}^{e}}(\tilde{A}) \rightarrow \operatorname{End}_{A_{C}^{e}}(\tilde{A})$. Using the explicit construction of internal Hom of DG-modules over a CDGA, it is straightforward to verify that $p^{*}$ is left inverse to $\iota$.

Next, consider the short exact sequence $F \rightarrow \tilde{A} \stackrel{\zeta}{\rightarrow} \Sigma_{2 n} \tilde{A}$. Applying $\operatorname{Hom}_{A_{C}^{e}}(-, \tilde{A})$ to this sequence yields a distinguished triangle of left $\operatorname{End}_{A_{C}^{e}}(\tilde{A})$-modules

$$
\Sigma^{2 n} \operatorname{End}_{A_{C}^{e}}(\tilde{A}) \stackrel{\zeta^{*}}{\rightarrow} \operatorname{End}_{A_{C}^{e}}(\tilde{A}) \stackrel{p^{*}}{\rightarrow} \operatorname{End}_{\tilde{A}_{B}^{e}}(\tilde{A}) .
$$

The morphism $\zeta^{*}: \Sigma^{2 n} \operatorname{End}_{A_{C}^{e}}(\tilde{A}) \rightarrow \operatorname{End}_{A_{C}^{e}}(\tilde{A})$ is just composition with $\zeta$, i.e., right multiplication by $\zeta \in \operatorname{End}_{A_{C}^{e}}(\tilde{A})$. This distinguished triangle yields a long exact sequence of homology groups. Since $H^{*}\left(p^{*}\right)$ is an epimorphism, we have a short exact sequence of graded left $R$-modules:

$$
\Sigma^{2 n} R \stackrel{\zeta}{\rightarrow} R \stackrel{H^{*}\left(p^{*}\right)}{\longrightarrow} Q .
$$

Note that there are two multiplicative structures on $Q$ : the usual one and the one coming from $Q$ being a quotient of the graded ring $R$ by the ideal $(\zeta)$. These structures must coincide, because $H^{*}(\iota)$ is multiplicative and has a left inverse.

The second ingredient is that the homotopy inverse limit of the tower

$$
\mathcal{T}:=[R \stackrel{z}{\leftarrow} R \stackrel{z}{\leftarrow} \cdots]
$$

is zero. Because $A$ is zero in negative codegrees, the homotopy colimit $\tilde{A}^{\infty}$ of the telescope $\tilde{A} \stackrel{\zeta}{\rightarrow} \Sigma_{2 n} \tilde{A} \stackrel{\zeta}{\rightarrow} \cdots$ is zero. Applying $\operatorname{Hom}_{A_{C}^{e}}(-, \tilde{A})$ to this telescope gives a tower

$$
\operatorname{End}_{A_{C}^{e}}(\tilde{A}) \stackrel{\xi^{*}}{\longleftarrow} \Sigma^{2 n} \operatorname{End}_{A_{C}^{e}}(\tilde{A}) \stackrel{\zeta^{*}}{\longleftarrow} \cdots .
$$

Its homotopy inverse limit, $\operatorname{Hom}_{A_{C}^{e}}\left(\tilde{A}^{\infty}, \tilde{A}\right)$ is therefore also zero. By the Milnor exact sequence, $\lim \mathcal{T}=0$ and $R^{1} \lim _{\leftarrow} \mathcal{T}=0$.

Next, consider the two towers:

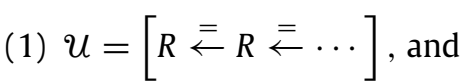

(2) $\mathcal{V}=\left[Q=R /(\zeta) \leftarrow R /\left(\zeta^{2}\right) \leftarrow R /\left(\zeta^{3}\right) \leftarrow \cdots\right]$.

There is a short exact sequence of towers $0 \rightarrow \mathcal{J} \rightarrow U \rightarrow \mathcal{V} \rightarrow 0$, given by:

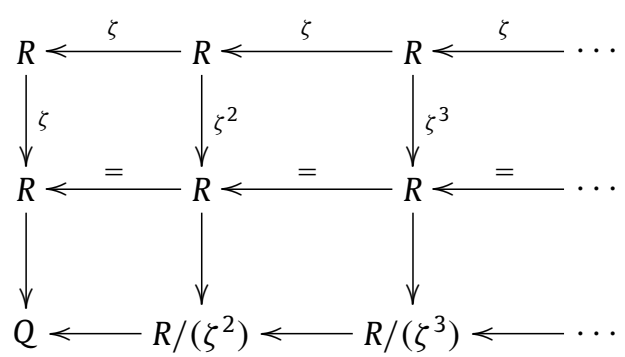

The six term exact sequence:

$$
0 \rightarrow \lim _{\leftarrow} \mathcal{T} \rightarrow \lim _{\leftarrow} u \rightarrow \lim _{\leftarrow} \mathcal{V} \rightarrow \lim _{\leftarrow}^{1} \mathcal{T} \rightarrow \lim _{\leftarrow}^{1} u \rightarrow \lim _{\leftarrow}^{1} \mathcal{V} \rightarrow 0
$$

shows that $R$ (which is isomorphic to $\lim _{\leftarrow} U$ ) is isomorphic to $\lim _{\leftarrow} \mathcal{V}$. 
To complete the proof we need to show that $R /\left(\zeta^{n}\right)$ is isomorphic to the truncated polynomial ring $Q[\zeta] /\left(\zeta^{n}\right)$. Observe that $\left(\zeta^{n}\right) /\left(\zeta^{n+1}\right)$ is isomorphic to $Q$ as an $R$-module, and that there is a subalgebra $Q[\zeta] \subseteq R$. These facts yield a morphism of short exact sequences:

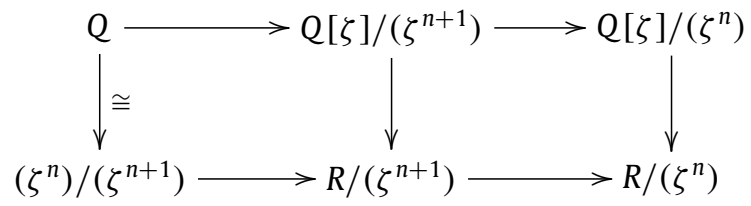

Since $R /(\zeta) \cong Q$ we get an inductive argument showing that $R /\left(\zeta^{n}\right) \cong Q[\zeta] /\left(\zeta^{n}\right)$ as rings.

Therefore

$$
R \cong \lim _{\leftarrow} Q[\zeta] /\left(\zeta^{n}\right)=Q[[\zeta]]
$$

as required.

\section{Polynomial growth implies spherical extension}

The purpose of the present section is to complete the loop of implications and show that the gci condition has a major structural implication. Unfortunately the Noetherian condition does not in itself have appropriate hereditary properties, so we are forced to impose the strongly Noetherian condition 3.5 .

Theorem 12.1. If $X$ is a strongly Noetherian gci space, it is also sci.

This states that a finiteness condition (strongly Noetherian and finite homotopy) implies that a space has a particular form (fibration $F \longrightarrow X \longrightarrow K V$, where $\pi_{*}(F)$ is in odd degrees) and is therefore perhaps the most interesting step.

\subsection{Strategy}

Assume $X$ is gci. By the Milnor-Moore theorem, $\pi_{*}(\Omega X)$ is finite dimensional, and in particular its even part is finite dimensional.

We argue by induction on $\operatorname{dim}_{\mathbb{Q}}\left(\pi_{*}(X)\right)$. The result is trivial if $\pi_{*}(X)=0$, and the inductive step will be to reduce the dimension of the top homotopy group and retain the Noetherian condition. Suppose then that the top non-zero homotopy is in degree $s$ and that $x$ is a non-zero element of the vector space dual $\pi_{s}^{\vee}(X)$.

If $s$ is odd ( $\operatorname{say} s=2 n-1$ ), killing homotopy groups gives a fibration

$$
S^{2 n-1} \longrightarrow X \longrightarrow X^{\prime}
$$

with $\operatorname{dim}_{\mathbb{Q}}\left(\pi_{*}\left(X^{\prime}\right)\right)=\operatorname{dim}_{\mathbb{Q}}\left(\pi_{*}(X)\right)-1$. By the strongly Noetherian condition, $X^{\prime}$ is also strongly Noetherian. Since the dimension of the dual homotopy groups of $X^{\prime}$ is finite, $X^{\prime}$ is gci, and by induction we conclude it is also sci. The fibration displays $X$ as being sci as required.

If $s$ is even ( say $s=2 n$ ), killing homotopy groups gives a fibration, $K(\mathbb{Q}, 2 n) \longrightarrow X \longrightarrow Y$, but this is not of use to us. We will argue, heavily using the fact that $H^{*}(X)$ is Noetherian, that in fact the element $x$ is in the image of the dual Hurewicz map; this is done in Proposition 12.2. Accordingly there is another fibration

$$
X^{\prime} \longrightarrow X \longrightarrow K(\mathbb{Q}, 2 n)
$$

where $\operatorname{dim}_{\mathbb{Q}}\left(\pi_{*}\left(X^{\prime}\right)\right)=\operatorname{dim}_{\mathbb{Q}}\left(\pi_{*}(X)\right)-1$. By taking an appropriate Postnikov section we see that both $K(Q, 2 n)$ and $X^{\prime}$ are retracts of $X$, and in particular $X^{\prime}$ is strongly Noetherian (in fact $X^{\prime} \simeq Y$ ). By induction we conclude $X^{\prime}$ is sci, and from Corollary 7.3 it follows that $X$ is sci.

\subsection{The dual Hurewicz map}

In rational homotopy it is natural to dualize the Hurewicz map

$$
h: \pi_{n}(X) \longrightarrow H_{n}(X)
$$

and concentrate on the dual Hurewicz map

$$
h^{\vee}: H^{*}(X)=H_{*}(X)^{\vee} \rightarrow \pi_{*}(X)^{\vee} .
$$

In fact, the dual Hurewicz map $h^{\vee}$ must be zero on decomposable elements of $H^{*} X$, and so it yields a map from the indecomposable quotient of $H^{*}(X)$ to $\pi_{*}(X)^{\vee}$. 
If $(\Lambda V, d)$ is a minimal Sullivan model for $X$ then this dual Hurewicz map is the linear map

$$
h^{\vee}: H^{*}(\Lambda V, d) \rightarrow V
$$

that comes from dividing $(\Lambda V, d)$ by the sub-cochain complex $\left(\Lambda^{\geq 2} V, d\right)$. An element $x \in V$ is in the image of $h^{\vee}$ if and only if there is a $g \in \Lambda^{\geq 2} V$ such that $d(x+g)=0$.

\subsection{The dual Hurewicz map and the Noetherian condition}

It is immediate from Theorem 7.1 that if $X$ is sci then $h^{\vee}$ is an epimorphism in even degrees. The critical step in showing that gci implies sci is to prove a special case of this surjectivity holds for gci spaces. We are grateful to $S$. Iyengar for pointing out that a corresponding result with a very different proof appears as a crucial lemma in [6].

Proposition 12.2. Suppose $X$ is a rational space with finite dimensional homotopy and that the top degree in which homotopy is nonzero is $2 n$. If $H^{*}(X)$ is Noetherian then the dual Hurewicz map

$$
h^{\vee}: H^{2 n}(X) \longrightarrow \pi_{2 n}^{\vee}(X)
$$

is surjective.

Proof. We choose an element $x$ of dual homotopy and show it lies in the image of the dual Hurewicz map.

By killing homotopy groups, there is a fibration sequence

$$
K(\mathbb{Q}, 2 n) \stackrel{\Phi}{\rightarrow} X \longrightarrow Y
$$

so that $\Phi$ is a monomorphism in homotopy. We suppose $S=(\Lambda W, d)$ is a minimal Sullivan model for $Y$, and $R=(\Lambda V, d)$ is a minimal Sullivan model for $X$, where $V=W \oplus \mathbb{Q} x$. We can take $Q=(\Lambda x, 0)$ as a Sullivan model for $K(\mathbb{Q}, 2 n)$ with $\Phi: R \rightarrow Q$ being the obvious map, so that

$$
S \rightarrow R \stackrel{\Phi}{\rightarrow} Q \simeq R \otimes_{S} \mathbb{Q}
$$

provides an algebraic model of the fibration.

We will show shortly that if $H^{*}(\Phi)=0$ then we reach a contradiction, and in view of the following lemma this will complete the argument.

Lemma 12.3. If $H^{*}(\Phi)$ is non-zero, then $x$ is in the image of the dual Hurewicz map.

Proof. Since $H^{*}(\Phi) \neq 0$ there exists an $i$ such that

$$
x^{i} \in \operatorname{im}\left(H^{*}(X) \rightarrow H^{*}(K(\mathbb{Q}, n))=\mathbb{Q}[x]\right) .
$$

This implies there is a cocycle in $R$ of the form:

$$
x^{i}+f_{1} x^{i-1}+f_{2} x^{i-2}+\cdots+f_{i}
$$

with $f_{j} \in \Lambda W$. The differential of this cocycle is

$$
\left(i d x+d f_{1}\right) x^{i-1}+[\text { terms of lower degree in } x]=0,
$$

which implies $d x+d f_{1} / i=0$. Now remark that $x+f_{1} / i$ cannot be a coboundary because $R$ is minimal, and hence $x$ is in the image of the dual Hurewicz map.

We shall next employ a functor on the derived category of $C^{*}(X)$ called $\mathbb{Q}$-cellular approximation. As explained in [7], this functor is well understood when $H^{*}(X)$ is Noetherian, and for this reason $\mathbb{Q}$-cellular approximation is useful to us. We begin by recalling the relevant definitions.

An object in the derived category of $C^{*}(X)$-modules is said to be $\mathbb{Q}$-cellular if it is built from $\mathbb{Q}$ up to equivalence. A map $M \longrightarrow N$ of $C^{*}(X)$-modules is a $\mathbb{Q}$-equivalence if

$$
\operatorname{Hom}_{C^{*}(X)}(\mathbb{Q}, M) \longrightarrow \operatorname{Hom}_{C^{*}(X)}(\mathbb{Q}, N)
$$

is a homology isomorphism. A map $M \longrightarrow N$ is $\mathbb{Q}$-cellular approximation if it is a $\mathbb{Q}$-equivalence and $M$ is $\mathbb{Q}$-cellular. By the usual formal argument, this is unique up to equivalence, and we write $\operatorname{Cell}_{\mathbb{Q}}(N) \longrightarrow N$ for it.

Cellularization has no effect on a trivial $R$-module (i.e., one pulled back from a $\mathbb{Q}$-module along the augmentation) in the sense that

$$
\operatorname{Cell}_{\mathbb{Q}}^{R}(M) \simeq M .
$$

Lemma 12.4. If $K(\mathbb{Q}, 2 n) \longrightarrow X \longrightarrow Y$ is a fibration sequence killing a top homotopy group of $X$ then $C^{*}(\Omega Y)$ is $\mathbb{Q}$-cellular as a module over $C^{*}(K(\mathbb{Q}, 2 n))$. 
Proof. Since both $\Omega X$ and $\Omega Y$ are products of Eilenberg-MacLane spaces, the connecting map $\Omega Y \longrightarrow K(\mathbb{Q}, 2 n)$ must be null. It follows that the induced map in cohomology $\mathbb{Q}[x]=H^{*}(K(\mathbb{Q}, 2 n)) \longrightarrow H^{*}(\Omega Y)$ factors through $\mathbb{Q}$, and hence that $C^{*}(\Omega Y)$ is $\mathbb{Q}$-cellular as required.

The lemma verifies the hypothesis of the Strong Independence of Base property $[18,3.1]$ for the map $R \rightarrow Q$ with $k=\mathbb{Q}$ and so we may conclude

$$
\operatorname{Cell}_{\mathbb{Q}}^{R}(Q) \simeq \operatorname{Cell}_{\mathbb{Q}}^{Q}(Q),
$$

and we will consider these two equivalent cellularizations in turn.

In favourable cases we may make calculations of the homology of cellularizations. If $H^{*}(X)$ is Noetherian, then by Dwyer et al. [7, 9.3], the stable Koszul complex can be used to construct the $\mathbb{Q}$-cellularization and there is a local cohomology spectral sequence

$$
H_{I}^{-p}\left(H^{*}(Q)\right)_{q} \Rightarrow H_{p+q}\left(\operatorname{Cell}_{\mathbb{Q}}^{R}(Q)\right),
$$

where $Q$ is considered as an $R$-module via $\Phi$.

Applying this to $\operatorname{Cell}_{\mathbb{Q}}^{Q}(Q)$, we see that since $H^{*}(Q)=\mathbb{Q}[x]$, the local cohomology spectral sequence collapses to show

$$
H^{*}\left(\operatorname{Cell}_{\mathbb{Q}}^{Q}(Q)\right)=\Sigma H_{I}^{1}(\mathbb{Q}[x])=\Sigma \mathbb{Q}[x]^{\vee} .
$$

In particular $\operatorname{Cell}_{\mathbb{Q}}^{Q} Q$ has cohomology in negative codegrees.

Turning to $\operatorname{Cell}_{\mathbb{Q}}^{R}(Q)$, we see that if $H^{*}(\Phi)=0$ we reach a contradiction. Indeed, in this case $Q$ is a trivial $R$-module and therefore Cell $\mathbb{Q}_{\mathbb{Q}}(Q) \simeq Q$. Since this is entirely in codegrees $\geq 0$ it contradicts the equivalence with Cell $\mathbb{Q}_{\mathbb{Q}}^{Q}(Q)$. Accordingly $H^{*}(\Phi)$ is non-zero and so Lemma 12.3 always applies, and this completes the proof of the proposition.

This completes the proof of Theorem 12.1.

\section{The evs condition}

In this section we conclude by giving a condition in the style of the zci condition which captures polynomial growth in the non-Noetherian situation. The idea of the condition is that it is a coherent version of virtual smallness with a similar flavour to the eci condition, so we call it the evs condition. From another point of view, since Example 8.6 shows that the Noetherian condition is essential, the evs condition introduced here is genuinely weaker than both the zci and eci conditions.

\subsection{The condition}

We suppose that $R$ is a CDGA and continue to write $\mathbf{D}(R)$ for the derived category of dg-R-modules.

Definition 13.1. We say that $R$ is evs of length $\leq n$ if there are

(1) a sequence of triangulated subcategories $\mathbf{D}(R)=\mathbf{D}_{0} \supseteq \mathbf{D}_{1} \supseteq \cdots \supseteq \mathbf{D}_{n}$ (not necessarily full) and

(2) natural transformations $\zeta_{i}: 1_{\mathbf{D}_{i}} \rightarrow \Sigma_{\left|\zeta_{i}\right|} 1_{\mathbf{D}_{i}}$ for $i=0, \ldots, n-1$

such that the following conditions hold.

(1) Every $\mathbf{D}_{i}$ is closed under coproducts.

(2) Every $\zeta_{i}$ is central among natural transformations of $\mathbf{1}_{\mathbf{D}_{i}}$.

(3) For every $X \in \mathbf{D}_{i}$ there exist an object $X / \zeta_{i} \in \mathbf{D}_{i+1}$ and a distinguished triangle $X \stackrel{\zeta_{i}}{\rightarrow} \Sigma_{\left|\zeta_{i}\right|} X \rightarrow X / \zeta_{i}$.

(4) If $0 \neq X \in \mathbf{D}_{i}$ is in the thick subcategory generated by $\mathbb{Q}$ then $X / \zeta_{i}$ is non-zero.

(5) If $0 \neq X \in \mathbf{D}(R)$ is in the thick subcategory generated by $\mathbb{Q}$ then $X / \zeta_{0} / \zeta_{1} / \cdots / \zeta_{n-1}$ is non-zero and small as an object of $\mathbf{D}(R)$.

Remark 13.2. (i) Note first that if $R$ is zci or eci of codimension $c$, it is clearly evs of length $c$.

(ii) On the other hand, if $R$ is evs and the natural transformations $\zeta_{1}, \ldots, \zeta_{n-1}$ can be extended to central natural transformations of $1_{\mathbf{D}(R)}$, then the condition is much like the zci condition without requiring that the cohomology be Noetherian. Remark 13.11 below provides an explicit example where it is not possible to extend one of these natural transformations.

(iii) In the evs condition there is no limitation on the degrees of the natural transformations $\zeta_{i}$. This explains why proving that evs implies polynomial growth (Lemma 13.5) is so much harder than proving the similar result for the eci condition.

In the context of rational homotopy theory there is a straightforward characterization of evs CDGAs.

Theorem 13.3. Let $R=(\Lambda V, d)$ be a minimal Sullivan algebra. Then $R$ is evs if and only if $V$ is finite dimensional.

Lemma 13.5 below shows that if $V$ is infinite dimensional, then $R$ is not evs. The converse is proved in Section 13.7 below.

Remark 13.4. The contrast with eci spaces, where Theorem 7.1 shows the structure is much more constrained (the differential on even generators is zero) is very striking.

On the other hand, the only difference is the Noetherian condition. Indeed, if $X$ is evs and strongly Noetherian, then Theorem 13.3 shows that $X$ is gci and therefore (by Theorems 11.1 and 12.1) also eci. 


\subsection{Polynomial growth}

The hard work in this section is in dealing with the case of a natural transformation of degree zero.

Lemma 13.5. Let $X$ be a simply-connected finite $C W$-complex. If $C^{*}(X)$ is evs, then $H_{*}(\Omega X)$ has polynomial growth.

Proof. Since $R=C^{*}(X)$ is evs there is a sequence of $R$-modules $M_{0}, M_{1}, \ldots, M_{n}$ such that the following hold.

(1) $M_{0}=\mathbb{Q}$ and $M_{i} \in \mathbf{D}_{i}$.

(2) There is a distinguished triangle $\Sigma^{\left|\zeta_{i}\right|} M_{i} \stackrel{\zeta_{i}}{\rightarrow} M_{i} \rightarrow M_{i+1}$.

(3) $M_{n}$ is a small $R$-module.

Suppose, by way of contradiction, that $H^{*}(\Omega X)$ has exponential growth. As in Section 9 , if all the degrees $\left|\zeta_{0}\right|, \ldots,\left|\zeta_{n-1}\right|$ are non-zero, then we have a contradiction, since by Lemma $9.3 H^{*}\left(M_{n} \otimes_{R} \mathbb{Q}\right)$ must have exponential growth because $H^{*}\left(M_{0} \otimes_{R} \mathbb{Q}\right) \cong H^{*}(\Omega X)$ has exponential growth.

We are left with showing that if $\left|\zeta_{i}\right|=0$ for some $i$, the exponential growth still propagates. So suppose that $\left|\zeta_{i}\right|=0$ for some $i$ and $M_{i} \otimes_{R} \mathbb{Q}$ has exponential growth. Consider the homotopy colimit $M_{i}^{\infty}$ of the telescope $M_{i} \stackrel{\zeta_{i}}{\rightarrow} M_{i} \stackrel{\zeta_{i}}{\rightarrow} \cdots$. Since this homotopy colimit is part of a distinguished triangle: $\oplus_{n=0}^{\infty} M_{i} \stackrel{1-\zeta_{i}}{\longrightarrow} \oplus_{n=0}^{\infty} M_{i} \rightarrow M_{i}^{\infty}$ where the first map is in $\mathbf{D}_{i}, M_{i}^{\infty}$ is also in $\mathbf{D}_{i}$.

Next we show that $M_{i}^{\infty}$ is finitely built from $\mathbb{Q}$. By construction $H^{*}\left(M_{i}\right)$ is non-zero only in finitely many degrees. Each cohomology group $H^{j}\left(M_{i}\right)$ is a finite dimensional vector space on which $\zeta_{i}$ acts as a linear transformation. For large enough $m$, the kernel of $\zeta_{i}^{m}$ stabilizes. Denote this kernel by $K \subseteq H^{j}\left(M_{i}\right)$, so that we can write

$$
H^{j}\left(M_{i}\right) \cong K \oplus V,
$$

where $\zeta_{i}^{m}$ is zero on $K$ and is an isomorphism on $V$. We see that $H^{j}\left(M_{i}^{\infty}\right) \cong V$ is also finite dimensional, and $M_{i}^{\infty}$ itself is finitely built from $\mathbb{Q}$.

The morphism $M_{i}^{\infty} \stackrel{\zeta_{i}}{\rightarrow} M_{i}^{\infty}$ is an equivalence, so Condition (4) of the definition of evs shows $M_{i}^{\infty} \simeq 0$. As $M_{i}^{\infty} \otimes_{R} \mathbb{Q} \simeq 0$ and $H^{j}\left(M_{i} \otimes_{R} \mathbb{Q}\right)$ is finite dimensional for each $j$, it follows that $H^{j}\left(\zeta_{i} \otimes_{R} \mathbb{Q}\right)$ is nilpotent on $H^{j}\left(M_{i} \otimes_{R} \mathbb{Q}\right)$ for each $j$, i.e., for each $j$ there exists an $n$ such that $\zeta_{i}^{n} \otimes_{R} \mathbb{Q}$ induces the zero map on $H^{j}\left(M_{i} \otimes_{R} \mathbb{Q}\right)$.

First observe that $\left[M_{i}, M_{i}\right]_{R}$ (i.e., the ring of degree 0 homotopy endomorphisms) is a finite dimensional algebra. Indeed, we start by observing that $\left[M_{0}, M_{0}\right]_{R}^{*}=[\mathbb{Q}, \mathbb{Q}]_{R}^{*}$ is finite dimensional in each degree, and deduce the same for $\left[M_{1}, M_{1}\right]_{R}^{*},\left[M_{2}, M_{2}\right]_{R}^{*}, \ldots,\left[M_{i}, M_{i}\right]_{R}^{*}$ using the defining triangles.

Now let $z$ be the morphism $M_{i} \otimes_{R} \mathbb{Q} \stackrel{\zeta_{i} \otimes_{R} \mathbb{Q}}{\longrightarrow} M_{i} \otimes_{R} \mathbb{Q}$, and note that the span of $\left\{z, z^{2}, z^{3} \ldots\right\}$ inside $\left[M_{i} \otimes_{R} \mathbb{Q}, M_{i} \otimes_{R}\right.$ $\mathbb{Q}]_{c_{*}(\Omega X)} \cong\left[M_{i}, M_{i}\right]_{R}$ is finite dimensional. We now resort to a classical trick to show that $z$ is nilpotent on $H^{*}\left(M_{i} \otimes_{R} \mathbb{Q}\right)$, i.e., that there is some $N$ for which $z^{N}$ induces the zero map on $H^{*}\left(M_{i} \otimes_{R} \mathbb{Q}\right)$.

Suppose $\left\{z, z^{2}, \ldots, z^{n}\right\}$ is a basis for the span of $\left\{z, z^{2}, z^{3}, \ldots\right\}$. Let $x \in H^{j}\left(M_{i} \otimes_{R} \mathbb{Q}\right)$ for some $j$ and let $\left\{z(x), z^{2}(x), \ldots, z^{k}(x)\right\}$ be a basis for the span of $\left\{z(x), z^{2}(x), z^{3}(x), \ldots\right\}$. Clearly $k \leq n$. Suppose that $z^{m}(x) \neq 0$ and $z^{m+1}(x)=0$, so that $m \geq k$. We will show that $m=k$ so $z^{k+1}(x)=0$.

Write

$$
z^{m}(x)=a_{1} z(x)+a_{2} z^{2}(x)+\cdots+a_{j} z^{j}(x),
$$

where $a_{j} \neq 0$ and $j \leq k$. First, we note that $j=k$. Indeed, applying $z$ we find $0=z^{m+1}(x)=\sum_{i=1}^{j} a_{i} z^{i+1}(x)$, so that if $j<k$ we get a linear dependence. Thus, for some $t \leq k$ we have

$$
z^{m}(x)=a_{t} z^{t}(x)+a_{t+1} z^{t+1}(x)+\cdots+a_{k} z^{k}(x),
$$

where $a_{t} \neq 0$. It suffices to show $t=k$, so we suppose $t<k$ and deduce a contradiction. If $t<k$ we apply $z$ to our equation and deduce

$$
z^{m+1}(x)=0=a_{t} z^{t+1}(x)+a_{t+1} z^{t+2}(x)+\cdots+a_{n-1} z^{k}(x)+a_{k} z^{k+1}(x)
$$

which means that $z^{k+1}(x)$ is in the span of $\left\{z^{t+1}(x), \ldots, z^{k}(x)\right\}$. Applying $z$ repeatedly, we deduce $z^{k+s}$ is also in the span of $\left\{z^{t+1}(x), \ldots, z^{k}(x)\right\}$ for all $s \geq 1$. Now either $m=k$ and we are done, or $m=k+s$ for some $s \geq 1$ and we obtain a contradiction. Accordingly, $t=k$ and $z^{k+1}(x)=0$ as required.

Since $H^{*}\left(M_{i} \otimes_{R} k\right)$ has exponential growth, it follows that the kernel of the map $H^{*}(z): H^{*}\left(M_{i} \otimes_{R} k\right) \rightarrow H^{*}\left(M_{i} \otimes_{R} k\right)$ has exponential growth. In particular, this implies that the cone of $z=\zeta_{i} \otimes \mathbb{Q}: M_{i} \otimes_{R} \mathbb{Q} \rightarrow M_{i} \otimes_{R} \mathbb{Q}$ has exponential growth. 


\subsection{The first unravelling move}

We now describe three constructions we may use to build a new evs space $X$ from a given evs space $X^{\prime}$. In practice we are given $X$, and we unravel the process to obtain $X^{\prime}$ in such a way that if $X^{\prime}$ is evs, so too is $X$. Only the last of these three was necessary in the eci case (it was the critical role of Proposition 12.2 to show this). We work entirely algebraically, so that $R$ is a model for $X$ and $R^{\prime}$ is a model for $X^{\prime}$.

The first move is eliminating an even generator that is also a cocycle.

Lemma 13.6. Let $R=(\Lambda V, d)$ be a minimal Sullivan algebra and let $x \in V$ be an element of even degree such that $d x=0$. Then multiplication by $x$ yields a natural transformation on $\mathbf{D}(R): M \stackrel{x \cdot}{\rightarrow} \Sigma^{-|x|} M$ whose cone is $R /(x) \otimes_{R} M$. If $R /(x)$ is evs of length $\leq n$ then $R$ is evs of length $\leq n+1$.

We record the topological counterpart of this lemma.

Lemma 13.7. Let $x \in \pi_{2 n}^{\vee}(X)$ be an element that is in the image of the dual Hurewicz map $H^{*}(X) \rightarrow \pi_{*}^{\vee}(X)$. Then there is a fibration sequence:

$$
X^{\prime} \rightarrow X \rightarrow K(\mathbb{Q} x)
$$

such that $x$ is in the image of $\pi_{2 n}^{\vee}(K(\mathbb{Q} x))$. If $X^{\prime}$ is evs of length $\leq n$ then $X$ is evs of length $\leq n+1$.

Proof of Lemma 13.6. In this case there is a short exact sequence of $\mathrm{dg}$ - $R$-modules:

$$
\Sigma^{|x|} R \hookrightarrow R \rightarrow R /(x) .
$$

The leftmost map is given by multiplication by $x: a \mapsto a \cdot x$. We write $V=W \oplus \mathbb{Q} x$ so that $R /(x) \cong(\Lambda W, d)$, isomorphic to a sub-DGA of $R$.

Define a natural transformation $\zeta: 1_{\mathbf{D}(R)} \rightarrow \Sigma^{-|x|} 1_{\mathbf{D}(R)}$ as multiplication by $x$. If $M$ is a cofibrant dg-R-module, then applying $-\otimes_{R} M$ to the short exact sequence above yields a distinguished triangle

$$
\Sigma^{|x|} M \stackrel{\zeta}{\rightarrow} M \rightarrow R /(x) \otimes_{R} M
$$

in $\mathbf{D}(R)$.

Now suppose that $R /(x)$ is evs of length $\leq n$, so that there are subcategories $\mathbf{D}_{R /(x)}=\mathbf{D}_{0} \supseteq \mathbf{D}_{1} \supseteq \cdots \supseteq \mathbf{D}_{n}$ and appropriate natural transformations $\zeta_{0}, \ldots, \zeta_{n-1}$. The map $p: R \rightarrow R /(x)$ of CDGAs induces an obvious functor $p^{*}: \mathbf{D}(R /(x)) \rightarrow \mathbf{D}(R)$. Define $\mathbf{D}_{i}^{\prime}=p^{*} \mathbf{D}_{i}$ and similarly $\zeta_{i}^{\prime}=p^{*} \zeta_{i}$. Set $\mathbf{D}_{-1}^{\prime}=\mathbf{D}(R)$ and let $\zeta_{-1}^{\prime}$ be the natural transformation $\zeta$ defined above.

We may check that the subcategories $\mathbf{D}_{-1}^{\prime}, \ldots, \mathbf{D}_{n-1}^{\prime}$ and natural transformations $\zeta_{-1}^{\prime}, \ldots, \zeta_{n-1}^{\prime}$ satisfy the conditions for being evs. One need only note three things. First, if $M$ is a small $\operatorname{dg}-R /(x)$-module then $p^{*} M$ is a small dg-R-module, because $R /(x)$ is a small $R$-module. Second, if $N$ is a dg-R-module finitely built by $\mathbb{Q}$, then so is $R /(x) \otimes_{R} N$. The reason is that $N$ is finitely built by $\mathbb{Q}$ over $R$ if and only if $H^{*}(N)$ is finite dimensional. Third, if $N \neq 0$ is finitely built by $\mathbb{Q}$, then $N / \zeta$ is not zero, because $|\zeta| \neq 0$ and so $\zeta$ cannot induce an isomorphism of $H^{*}(N)$.

\subsection{The second unravelling move}

The second move eliminates an even cocycle by adding an odd generator. The argument is essentially the same as the previous one (except that the cocycle is not a generator), so we omit the proof.

Lemma 13.8. Let $R=(\Lambda V, d)$ be a minimal Sullivan algebra and let $f \in R$ be an even cocycle. Let $R^{\prime}=\left(\Lambda(V \oplus \mathbb{Q} y)\right.$, $\left.d^{\prime}\right)$, where $d^{\prime} y=f$ and $d^{\prime} v=d v$ for all $v \in V$. Then multiplication by $f$ yields a natural transformation on $\mathbf{D}(R): M \stackrel{f}{\rightarrow} \Sigma^{-|f|} M$ whose cone is $R^{\prime} \otimes_{R}$ M. If $R^{\prime}$ is evs of length $\leq n$ then $R$ is evs of length $\leq n+1$.

The topological counterpart of this lemma is again a fibration:

$$
X^{\prime} \rightarrow X \rightarrow K(\mathbb{Q} f),
$$

only this time we just require that $X \rightarrow K(\mathbb{Q} f)$ represent a nontrivial element in $H^{*}(X)$.

\subsection{The third unravelling move}

Finally, the third move is passing to a subalgebra. This is the precise counterpart of the argument of Section 12.1, and the conclusion is analogous to a spherical fibration

$$
S^{|x|-1} \longrightarrow X \longrightarrow X^{\prime}
$$

Nevertheless, we describe how this move fits within the evs context. 
Lemma 13.9. Let $R=(\Lambda V, d)$ be a minimal Sullivan algebra. Let $x \in V$ be an element of odd degree such that $d v \notin x \Lambda V$ for all $v \in V$. Then there are a sub Sullivan algebra $Q \subset R$ and a natural transformation $\zeta: 1_{\mathbf{D}(R)} \rightarrow \Sigma^{|x|+1} 1_{\mathbf{D}(R)}$ such that for any $M \in \mathbf{D}(R)$ there is a distinguished triangle in $\mathbf{D}(R)$ :

$$
M \stackrel{\zeta}{\rightarrow} \Sigma^{|x|+1} M \rightarrow \Sigma R \otimes_{\mathbb{Q}} M .
$$

If $Q$ is evs of length $\leq n$ then $R$ is evs of length $\leq n+1$.

Proof. Write $V=W \oplus \mathbb{Q} x$, and let $Q$ be the Sullivan algebra $(\Lambda W, d)$. Clearly $Q$ is a minimal Sullivan algebra and there is an obvious inclusion $\iota: Q \hookrightarrow R$ of Sullivan algebras. We have seen earlier that there is a natural transformation $\zeta$ on $\mathbf{D}(R)$ such that $M \stackrel{\zeta}{\rightarrow} \Sigma^{|x|+1} M \rightarrow \Sigma R \otimes_{\mathrm{Q}} M$ is a distinguished triangle for all $M \in \mathbf{D}(R)$.

Suppose that $Q$ is evs of length $\leq n$, so there are subcategories $\mathbf{D}_{Q}=\mathbf{D}_{0} \supseteq \mathbf{D}_{1} \supseteq \cdots \supseteq \mathbf{D}_{n}$ and appropriate natural transformations $\zeta_{0}, \ldots, \zeta_{n-1}$. The map $\iota: Q \rightarrow R$ of CDGAs induces a functor $\iota_{*}: \mathbf{D}(Q) \rightarrow \mathbf{D}(R)$, where $\iota_{*}(N)$ is the induced dg- $R$-module $R \otimes_{Q} N$. Define $\mathbf{D}_{i}^{\prime}=\iota_{*} \mathbf{D}_{i}$ and similarly $\zeta_{i}^{\prime}=\iota_{*} \zeta_{i}$. Set $\mathbf{D}_{-1}^{\prime}=\mathbf{D}(R)$ and let $\zeta_{-1}^{\prime}$ be the natural transformation $\zeta$ on $\mathbf{D}(R)$ defined above.

We may check that the subcategories $\mathbf{D}_{-1}^{\prime}, \ldots, \mathbf{D}_{n-1}^{\prime}$ and natural transformations $\zeta_{-1}^{\prime}, \ldots, \zeta_{n-1}^{\prime}$ satisfy the conditions for being evs. One need only note three things. First, if $N$ is a small DG- $Q$-module then $\iota_{*} N$ is a small DG- $R$-module. Second, if $M$ is a dg-R-module finitely built by $\mathbb{Q}$, then $M$ is finitely built by $\mathbb{Q}$ also as a dg- $Q$-module. Third, $|\zeta| \neq 0$ and therefore $M / \zeta \neq 0$ for every non-zero dg- $R$-module $M$ that is finitely built by $\mathbb{Q}$.

\subsection{Two examples}

We discuss some examples of minimal Sullivan algebras which are evs but not eci.

Example 13.10 ([10, Example 3.3(1)]). Consider the minimal Sullivan algebra

$$
R=\left(\Lambda\left(x_{3}, y_{3}, z_{3}, a_{8}\right), d x=d y=d z=0, d a=x y z\right) .
$$

First, we see that it is evs by showing explicitly how to unravel it. Indeed, we may apply Lemma 13.8 to the cocycle $x y$ to yield

$$
R^{\prime}=(\Lambda(x, y, z, w, a), d a=x y z, d w=x y) .
$$

Now, $d(w z)=d a$, so by a change of variables $a^{\prime}=a-w z$ we see that

$$
R^{\prime} \cong\left(\Lambda\left(x, y, z, w, a^{\prime}\right), d a^{\prime}=0, d w=x y\right) .
$$

Now $R^{\prime}$ is eci and from its homotopy we see it is of codimension 4. It is therefore also evs of length 4 , and therefore $R$ is evs of length $\leq 5$.

On the other hand, it is not hard to see that $R$ is not eci. Most explicitly, one may identify the cohomology ring explicitly and observe that it is not Noetherian: it has a basis of monomials $x^{i} y^{j} z^{k} a^{l}$ where $i, j, k \in\{0,1\}$ omitting the monomials $x y z a^{l}$ for $l \geq 0$ and $a^{l}$ for $l \geq 1$. All elements (except those in codegree zero) are nilpotent.

Note also that the dual Hurewicz map is not surjective in codegree 8, so that the method of Section 12.1 cannot be applied.

Remark 13.11. Finally, we can see explicitly why the natural transformation in the evs definition cannot always be extended as we would require for the zci definition. In the previous example, multiplication by the cocycle $a^{\prime}=a-w z$ defines a natural transformation on the $\mathbf{D}\left(R^{\prime}\right)$ and therefore also on the image of $\mathbf{D}\left(R^{\prime}\right)$ under restriction. This natural transformation cannot be extended to a central natural transformation of $1_{\mathbf{D}(R)}$, since we would then have a commutative diagram

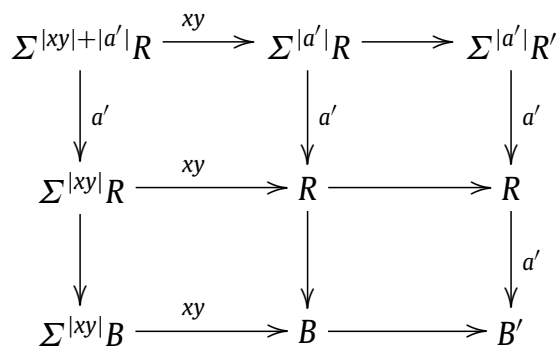

of distinguished triangles in $\mathbf{D}(R)$. The natural transformation $a^{\prime}$ must be the zero map on $R$, hence $B \cong R \oplus \Sigma^{|a|} R$. This shows that $B^{\prime}$ is isomorphic to $R^{\prime} \oplus \Sigma^{|a|} R^{\prime}$, and therefore $a^{\prime}$ acts as a regular element. However this contradicts the fact that $\left(a^{\prime}\right)^{2}=0$ from the long exact sequence of the triangle. 
Example 13.12. Consider the minimal Sullivan algebra

$$
R=\left(\Lambda\left(x_{5}, y_{3}, z_{3}, y_{3}^{\prime}, z_{3}^{\prime}, a_{10}\right), d x=y z+y^{\prime} z^{\prime}, d a=x y y^{\prime}\right)
$$

First, we see that it is evs by showing explicitly how to unravel it. We apply Lemma 13.8 to the cocycle $y y^{\prime}$, yielding:

$$
R^{\prime}=\left(\Lambda\left(x_{5}, y_{3}, z_{3}, y_{3}^{\prime}, z_{3}^{\prime}, a_{10}, w_{5}\right), d x=y z+y^{\prime} z^{\prime}, d a=x y y^{\prime}, d w=y y^{\prime}\right) .
$$

This yields $d(a+x w)=(d x) w$. We apply Lemma 13.8 twice more, for the cocycles $w y$ and $w y^{\prime}$, yielding:

$$
R^{\prime \prime}=\left(\Lambda\left(x_{5}, y_{3}, z_{3}, y_{3}^{\prime}, z_{3}^{\prime}, a_{10}, w_{5}, t_{7}, t_{7}^{\prime}\right), d x=y z+y^{\prime} z^{\prime}, d a=x y y^{\prime}, d w=y y^{\prime}, d t=w y, d t^{\prime}=w y^{\prime}\right) .
$$

Finally we have $d\left(a+x w-z t-z t^{\prime}\right)=0$. So, as in the previous example, we can do a change of variables $a^{\prime}=a+x w-z t-z t^{\prime}$ and see that $R^{\prime \prime}$ is eci of codimension 8 , and hence evs of length 8 . It follows that $R$ is evs of length $\leq 11$.

On the other hand, it is not hard to see that $R$ is not eci. Indeed, since the differential is non-zero on the top even class $a_{10}$, the dual Hurewicz map is not surjective in codegree 10, so that the method of Section 12.1 cannot be applied. By Proposition $12.2, H^{*}(R)$ is not Noetherian and so $R$ is not eci.

\subsection{Proof of Theorem 13.3}

Let $R=(\Lambda V, d)$ be a simply-connected minimal Sullivan algebra where $V$ is finite dimensional. We will show that $R$ is evs. The proof proceeds by induction on the dimension of $V^{\text {even }}$. The induction starts since if $V^{\text {even }}=0$, successive applications of the third unravelling move (Lemma 13.9) will reduce to a Sullivan algebra with trivial differential. This is then the model of a product of odd spheres, which is obviously evs.

If $V^{\text {even }} \neq 0$, then we apply Lemma 13.14 below, which says we may repeatedly apply the second unravelling move (i.e., add a finite number of odd generators) until we reach a CDGA $R^{\prime}$ with an even generator $a \in V^{\text {even }}$ such that $d a=0$. Now use the first unravelling move (Lemma 13.6) on $a$. The minimal Sullivan algebra $R^{\prime} /(a)$ is evs by the inductive hypothesis, so that $R^{\prime}$ is evs by Lemma 13.6, and $R$ is evs by Lemma 13.8 .

The key ingredient is the following technical result. Note that for a minimal Sullivan algebra $R=(\Lambda V, d)$ with $V$ of finite type, the image of an element $[f] \in H^{n}(R)$ under the dual Hurewicz $h^{\vee}$ map is non-zero if and only if there is an isomorphism of minimal Sullivan algebras $\rho: R \stackrel{\cong}{\rightarrow}\left(\Lambda V^{\prime}, d^{\prime}\right)$ such that $\rho(f) \in V^{\prime}$.

Lemma 13.13. Let $R=(\Lambda V, d)$ be a minimal (simply connected) Sullivan algebra such that $V$ is finite dimensional and concentrated only in odd degrees. Let $0 \neq[f] \in H^{2 i+1}(R)$ be an odd element of the cohomology of $R$. If $h^{\vee}([f])=0$, then there is an element $0 \neq[g] \in H^{2 j}(R)$ such that $0<j \leq i$.

Proof. Suppose the image of $[f]$ under the dual Hurewicz map is zero. The proof goes by induction on the dimension of $V$. Let $x \in V$ be an element of minimal codegree, therefore $d x=0$ and $h^{\vee}(x) \neq 0$. Eliminate $x$ by adding an even generator $S=(\Lambda(V \oplus \mathbb{Q} a), d a=x)$ ( $d$ being defined on $V$ as before). There is a distinguished triangle in $\mathbf{D}(R)$ :

$$
\Sigma^{|x|} S \rightarrow R \stackrel{\varphi}{\rightarrow} S \stackrel{\psi}{\rightarrow} \Sigma^{|x|+1} S .
$$

Since $S$ is equivalent to the minimal Sullivan algebra $R /(x)$, the induction assumption holds for $S$. There are two possible cases.

In the first case $H_{*} \varphi[f] \neq 0$. The image of $\varphi[f]$ under the dual Hurewicz map is zero because $\pi_{*}^{\vee} R \rightarrow \pi_{*}^{\vee} S$ is an epimorphism with kernel $\mathbb{Q} x$. By the induction assumption there is an even degree element in the cohomology of $S$ whose codegree is smaller than $|f|$. Let $[g]$ be such an element of minimal degree. If $[g]$ is in the image of $\varphi$, then we are done. If not, then $\psi[g] \neq 0$. But $\psi[g] \in H^{|g|-|x|+1}(S)$, which contradicts the minimality of $|g|$ (note that $|g|-|x|+1>0$, since otherwise $g$ is one degree below the minimal generators, which is impossible).

The remaining option is that $\varphi[f]=0$. Hence $f=d w$ for some $w \in S$. Write $w$ as

$$
w=a^{n} A_{n}+a^{n-1} A_{n-1}+\cdots+a A_{1}+A_{0},
$$

where $A_{i} \in \Lambda \mathrm{V}$. Clearly $f$ is homologous to $f-d A_{0}$, so without loss of generality we can assume that $A_{0}=0$. Also note that all the $A_{i}$ are of even codegrees smaller than the codegree of $f$ (in fact $\left|A_{i}\right| \leq|f|-|x|$ ). Calculating $d w$ gives

$$
d w=a^{n} d\left(A_{n}\right)+\sum_{i=1}^{n} a^{i-1}\left(d A_{i-1}+i x A_{i}\right) .
$$

Since $f \in \Lambda V$ we see that:

$$
\begin{aligned}
f & =x A_{1} \\
d A_{i-1} & =-i x A_{i} \quad \text { for } i \geq 2 \\
d A_{n} & =0 .
\end{aligned}
$$


Thus $A_{n}$ is a cocycle. If $A_{n}$ is not a coboundary in $R$, then we are done. Otherwise there is a $B_{n}$ so that

$$
A_{n}=d B_{n} \text {. }
$$

Now $d A_{n-1}=-n x A_{n}=-n x d B_{n}=d\left(n x B_{n}\right)$, whence $A_{n-1}-n x B_{n}$ is an even codegree cocycle. Again, if it is not a coboundary then we are done. Otherwise there is a $B_{n-1}$ so that

$$
A_{n-1}-n \times B_{n}=d B_{n-1}
$$

Now

$$
\begin{aligned}
d A_{n-2} & =-(n-1) x A_{n-1}=-(n-1) x\left(n x B_{n}+d B_{n-1}\right) \\
& =-(n-1) x d B_{n-1}=(n-1) d\left(x B_{n-1}\right) .
\end{aligned}
$$

So we see that $A_{n-2}-(n-1) x B_{n-1}$ is an even codegree cocycle. We continue in this manner until either we get the desired even codegree element in the cohomology of $R$, or we end with

$$
A_{1}-2 x B_{2}=d B_{1} \text {. }
$$

But now $f=x A_{1}=x\left(2 x B_{2}+d B_{1}\right)=x d B_{1}=-d\left(x B_{1}\right)$, i.e. $[f]=0$, which is a contradiction.

Using the previous lemma we can now show that the second unravelling move may be used to make an even generator become a cycle.

Lemma 13.14. Let $R=(\Lambda V, d)$ be a minimal (simply connected) Sullivan algebra such that $V$ is finite dimensional. Then there is a sequence $R=R_{0} \rightarrow R_{1} \rightarrow \cdots \rightarrow R_{n}$ of unravelling moves of the second type (i.e., moves to which Lemma 13.8 applies) such that $R_{n}$ is isomorphic to a minimal Sullivan algebra: $R_{n}^{\prime}=\left(\Lambda V^{\prime}, \delta\right)$, where there is a minimal even element a $\in V^{\prime}$ such that $\delta a=0$.

Proof. If there is a minimal even codegree element $a \in V$ such that $d a=0$ we are done. Otherwise, choose some minimal even codegree element $a \in V$. Let $V_{s} \subset V$ be the subspace of codegrees smaller than $\|a\|$. Minimality of the Sullivan algebra implies that $d a \in \Lambda V_{s}$.

Apply the second unravelling move (Lemma 13.8) to the even elements of $H^{*}(R)$ starting from the bottom and going up. Hence, let $g \in R$ be a minimal even degree cocycle that is not a coboundary. Applying Lemma 13.8 to $g$ yields

$$
R_{1}=\left(\Lambda\left(V \oplus \mathbb{Q} x_{g}\right), d x_{g}=g\right)
$$

and a map $R \rightarrow R_{1}$. We continue adding odd generators to $R$ in this way until there is no more homology in even codegrees less than or equal to $\|a\|$. Note that we need only kill cocycles composed of odd generators only, even in codegree $a$.

We end with a minimal Sullivan algebra $R_{n}=(\Lambda U, d)$, where $R_{n}$ has no even degree cohomology in dimension $\|a\|$ or lower. Let $U_{s} \subset U$ be the subspace of codegrees smaller than $\|a\|$. Clearly $U_{s}$ has only odd degree elements. Define $T=\left(\Lambda U_{s}, d\right)$ to be the appropriate sub-CDGA of $R_{n}$. Then $T$ also has no cohomology in even codegrees $\|a\|$ or lower. Because $d a$ is a cocycle in $T, h^{\vee}(d a)=0$ and $H^{2 j}(T)=0$ for $2 j \leq\|a\|$, it follows from Lemma 13.13 that $d a$ is a coboundary in $T$. Thus $d a=d u$ for some $u \in \Lambda U_{s}$.

Now $a-u$ is a cocycle in $R_{n}$. So a change of variables: $a^{\prime}=a-u$ yields a new minimal Sullivan algebra $R_{n}^{\prime}=\left(\Lambda U^{\prime}, \delta\right)$ where a minimal codegree even generator $a^{\prime}$ is a cocycle.

\section{Acknowledgement}

This work was supported by EPSRC Grant number EP/E012957/1.

\section{References}

[1] L.L. Avramov, Infinite free resolutions, in: Six Lectures on Commutative Algebra, Bellaterra, 1996, in: Progr. Math., vol. 166, Birkhäuser, Basel, 1998, pp. 1-118.

[2] L.L. Avramov, Locally complete intersection homomorphisms and a conjecture of Quillen on the vanishing of cotangent homology, Ann. Math. (2) 150 (2) (1999) 455-487.

[3] L.L. Avramov, R.-O. Buchweitz, Homological algebra modulo a regular sequence with special attention to codimension two, J. Algebra 230 (1) (2000) 24-67.

[4] L.L. Avramov, S. Halperin, Through the looking glass: a dictionary between rational homotopy theory and local algebra, in: Algebra, Algebraic Topology and their Interactions, Stockholm, 1983, in: Lecture Notes in Math., 1183, Springer-Verlag, 1986, pp. 1-27.

[5] D.J. Benson, J.P.C. Greenlees, S. Shamir, Complete intersections and mod p-cochain algebras, 2008. Preprint.

[6] N. Dupont, M. Vigué-Poirrier, Finiteness Conditions for Hochschild Homology Algebra and Free Loop Space Cohomology Algebra, K-Theory 21 (2000) 293300.

[7] W.G. Dwyer, J.P.C. Greenlees, S. Iyengar, Duality in algebra and topology, Adv. Math. 200 (2) (2006) 357-402.

[8] W.G. Dwyer, J.P.C. Greenlees, S. Iyengar, Finiteness in derived categories of local rings, Comment. Math. Helv. 81 (2) (2006) 383-432.

[9] D. Eisenbud, Homological algebra on a complete intersection, with an application to group representations, TAMS 260 (1980) 35-64.

[10] Y. Félix, S. Halperin, J.-C. Thomas, Gorenstein spaces, Adv. Math. 71 (1988) 92-112.

[11] Y. Félix, S. Halperin, J.-C. Thomas, Rational homotopy theory, in: Graduate Texts in Mathematics, vol. 205, Springer-Verlag, New York, ISBN: 0-38795068-0, 2001, xxxiv+535 pp.

[12] T.H. Gulliksen, On the deviations of a local ring, Math. Scand. 47 (1980) 5-20. 
[13] M.A. Mandell, $E_{\infty}$ algebras and p-adic homotopy theory, Topology 40 (1) (2001) 43-94.

[14] H. Matsumura, Commutative Ring Theory, CUP, 1986.

[15] J. Milnor, J.C. Moore, On the structure of Hopf algebras, Ann. Math. (2) 81 (1965) 211-264

[16] T Miller, J. Neisendorfer, Formal and coformal spaces, Illinois J. Math. 22 (1978) 565-580.

[17] J. Shamash, The Poincaré series of a local ring, J. Algebra 12 (1969) 453-470.

[18] S. Shamir, Cellular approximations and the Eilenberg-Moore spectral-sequence, Algebr. Geom. Topol. 9 (2009) 1309-1340. http://dx.doi.org/10.2140/ agt.2009.9.1309. 